\title{
High-precision Dynamical Masses of Pre-main-sequence Stars with ALMA and Gaia
}

\author{
Patrick D. Sheehan ${ }^{1,2}$ (1) , Ya-Lin Wu ${ }^{3,5}$ (1) Josh A. Eisner ${ }^{4}$ (1), and John J. Tobin ${ }^{1,2}$ (1) \\ ${ }^{1}$ National Radio Astronomy Observatory, 520 Edgemont Rd., Charlottesville, VA 22901, USA \\ ${ }^{2}$ Homer L. Dodge Department of Physics \& Astronomy, University of Oklahoma, 440 W. Brooks St., Norman, OK 73021, USA \\ ${ }^{3}$ McDonald Observatory and the Department of Astronomy, University of Texas at Austin, Austin, TX 78712, USA \\ ${ }^{4}$ University of Arizona Department of Astronomy and Steward Observatory, 933 North Cherry Ave., Tucson, AZ 85721, USA \\ Received 2018 October 31; revised 2019 February 20; accepted 2019 February 21; published 2019 April 1
}

\begin{abstract}
The Keplerian rotation in protoplanetary disks can be used to robustly measure stellar masses at very high precision if the source distance is known. We present Atacama Large Millimeter/submillimeter Array (ALMA) observations of spatially and spectrally resolved ${ }^{12} \mathrm{CO}(2-1)$ emission toward the disks around 2MASS J16262774-2527247 (the tertiary companion to ROXs 12 at $5100 \mathrm{au}$ ), CT Cha, and DH Tau. We employ detailed modeling of the Keplerian rotation profile, coupled with accurate distances from Gaia, to directly measure the stellar masses with $\sim 2 \%$ precision. We also compare these direct mass measurements with the masses inferred from evolutionary models, determined in a statistically rigorous way. We find that 2MASS J16262774-2527247 has a mass of $0.535_{-0.007}^{+0.006} M_{\odot}$ and CT Cha has a mass of $0.796_{-0.014}^{+0.015} M_{\odot}$, broadly consistent with evolutionary models, although potentially significant differences remain. $\mathrm{DH}$ Tau has a mass of $0.101_{-0.003}^{+0.004} M_{\odot}$, but it suffers from strong foreground absorption that may affect our mass estimate. The combination of ALMA, Gaia, and codes like pdspy, presented here, can be used to infer the dynamical masses for large samples of young stars and substellar objects, and place constraints on evolutionary models.
\end{abstract}

Key words: parallaxes - protoplanetary disks - radio lines: stars - stars: fundamental parameters - stars: pre-main sequence

\section{Introduction}

Protoplanetary disks are found nearly ubiquitously around pre-main-sequence stars (e.g., Hernández et al. 2008), and studies of these disks are crucial to understanding the environment in which stars and planets form. Now, thanks to the revolutionary power of the Atacama Large Millimeter/ submillimeter Array (ALMA), these disks have been studied in great detail. One notable development has been the unprecedented sensitivity to molecular line emission, which was inaccessible to previous generations of millimeter interferometers for all but the brightest sources (e.g., Simon et al. 2000). Access to gas emission has opened up a number of new avenues of study of protoplanetary disks, including their chemistry (e.g., Öberg et al. 2015; Schwarz et al. 2016), direct measurements of gas disk sizes and masses (e.g., Williams \& Best 2014; Ansdell et al. 2016, 2018; Miotello et al. 2017), as well as constraints on the turbulence in disks (Flaherty et al. 2015, 2017, 2018).

While there has been a significant effort toward studies of disk structure, the detection of gas emission has also created an opportunity to study the central star. Detailed radiative transfer modeling of the Keplerian motion in spatially and spectrally resolved disks has long been used as a tool for directly measuring stellar masses (e.g., Dutrey et al. 1994; Simon et al. 2000; Dutrey et al. 2003; Czekala et al. 2015, 2016, 2017; MacGregor et al. 2017; Ricci et al. 2017; Simon et al. 2017; Wu \& Sheehan 2017). However, the precision of these measurements is limited almost entirely by the uncertainty in the distance to the star, typically $>10 \%$, thereby limiting their usefulness for constraining evolutionary models. Although VLBI has produced precise distance measurements for some sources, potentially enabling precise mass measurements (e.g., Simon et al. 2017), care must be taken when extrapolating those distances (and

\footnotetext{
${ }^{5} 51$ Pegasi b Fellow.
}

distance uncertainties) to the star-forming region as a whole (e.g., Ortiz-León et al. 2017). In the era of precision distance measurements with Gaia, however, dynamical masses should be measurable with significantly improved precision for large samples of pre-main-sequence stars.

This development comes at a welcome time as, while theoretical evolutionary tracks have progressed significantly over the last few decades, major discrepancies remain between the models and the direct measurements of stellar radii and masses (Hillenbrand \& White 2004; Gennaro et al. 2012; Stassun et al. 2014; Bell 2016). Up to this point, constraints on pre-mainsequence tracks have primarily come from eclipsing binaries; however, these systems are relatively rare, with only $\sim 20$ having accurate mass measurements (e.g., Stassun et al. 2014). Astrometry of young binary systems provides another avenue for measuring masses (e.g., Dupuy et al. 2016; Rizzuto et al. 2016; Rodet et al. 2018), but requires precise measurements over a number of epochs. These measurements are also limited, by definition, to binary stars. On the contrary, many (or most) studies of young stars focus on single stars, which are easier to study. While it is typically assumed that relations between mass and temperature, radius, or other stellar parameters are common for binary and single stars, we must actually measure masses for a sample of single stars to test this assumption.

Constraints on evolutionary tracks are important for understanding fundamental stellar parameters (e.g., Stassun et al. 2014; Bell 2016), but also because such models are often used to draw conclusions about the star and planet formation processes. Cluster ages inferred from evolutionary tracks have been used to place constraints on protoplanetary disk lifetimes (e.g., Haisch et al. 2001; Hernández et al. 2008). More recently, a large amount of effort has gone into studying the disk-mass to stellar mass relation, how it evolves with time, and what that evolution means for disk evolution and planet formation (e.g., 

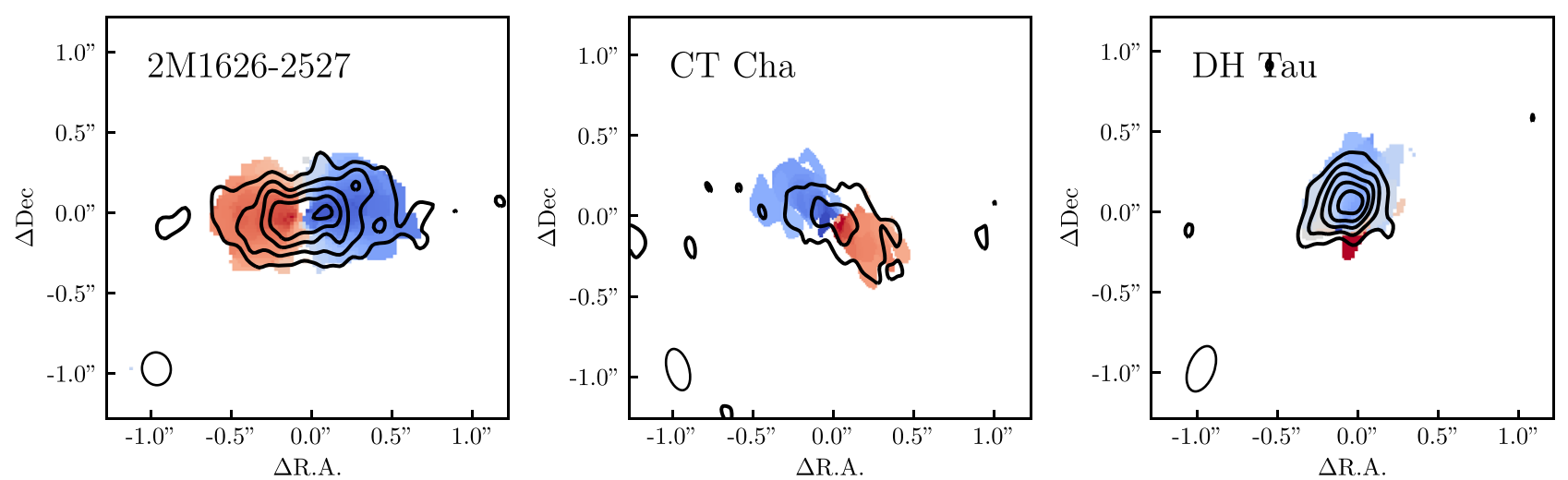

Figure 1. CO (2-1) moment maps for all three of our sources. We show the moment 0 (integrated intensity) map as black contours and the moment 1 velocity map as a color image. Contours of the moment 0 map start at $2 \sigma$ and subsequent contours are in intervals of $3 \sigma$. DH Tau shows a notable lack of redshifted emission, likely due to extinction by foreground clouds.

Table 1

Log of ALMA Observations

\begin{tabular}{|c|c|c|c|c|c|c|}
\hline Source & $\begin{array}{l}\text { Observation Date } \\
\text { (UT) }\end{array}$ & $\begin{array}{l}\text { Baselines } \\
(\mathrm{m})\end{array}$ & $\begin{array}{l}\text { Integration Time } \\
\text { (minutes) }\end{array}$ & Beam & $\begin{array}{l}\text { Rms } \\
(\mathrm{mJy})\end{array}$ & $\begin{array}{c}\text { Calibrators } \\
\text { (Flux/Bandpass, Gain) }\end{array}$ \\
\hline DH Tau & 2016 Sep 14 & $15-3247$ & 13 & $0 . " 30 \times 0$ ". 17 & 4 & $\mathrm{~J} 0510+1800, \mathrm{~J} 0433+2905$ \\
\hline 2M1626-2527 & 2016 Sep 16 & $15-3143$ & 12 & $0 ! " 21 \times 0$ "! 18 & 3.1 & $\mathrm{~J} 1517-2422, \mathrm{~J} 1634-2058$ \\
\hline CT Cha & 2016 Sep 27 & $15-3247$ & 13.5 & $0 ! .27 \times 0 !^{\prime \prime} 14$ & 3.8 & $\mathrm{~J} 1107-4449, \mathrm{~J} 1058-8003$ \\
\hline
\end{tabular}

Andrews et al. 2013; Ansdell et al. 2016; Barenfeld et al. 2016; Pascucci et al. 2016; Eisner et al. 2018). However, if stellar properties are derived from pre-main-sequence evolutionary tracks, they are always model-dependent (e.g., Andrews et al. 2013; Ward-Duong et al. 2018).

Here we demonstrate how ALMA ${ }^{12} \mathrm{CO}(2-1)$ observations and precise distances with Gaia can be used to measure high-precision ( $\sim 2 \%$ ) dynamical masses for young stars, using observations of the three pre-main-sequence stars 2MASS J16262774-2527247, CT Cha, and DH Tau. These sources were originally observed as part of a program to look for disks around young, accreting planetary-mass companions in both continuum and spectral line emission. The results of this program as it pertains to the companions are presented in $\mathrm{Wu}$ et al. (2017b), Wu \& Sheehan (2017), and $\mathrm{Wu}$ et al. (2017a). The star 2MASS J16262774-2527247 (hereafter 2M1626-2527) was observed due to a mix-up propagated through the literature that identified its coordinates as those of the $\sim 17 M_{\text {Jup }}$ planetary-mass companion ROXs 12 B (Bowler et al. 2017). Interestingly, 2M1626-2527 is also shown to be a companion to ROXs 12 A (Bowler et al. 2017). Although these sources are not truly "single," this technique can be equally well applied to any star, single or multiple, with a disk.

The paper is structured as follows. In Section 2 we describe the ALMA observations of our three targets. In Section 3.1 we present our modeling analysis of the spectral line observations, including their mass measurements, using our new code pdspy $^{6}$ (Sheehan 2018). Then, in Section 3.2, we use theoretical evolutionary tracks to calculate stellar masses for our sources, and compare with our dynamically measured masses in Sections 4 and 5. Finally, we provide extensive appendices to further document the modeling technique as well as the pdspy code. pdspy can handle both spectral line and

$\overline{6}$ The pdspy code can be found here: https://github.com/psheehan/pdspy. continuum observations, and is now publicly available. The continuum portion of the code was used in Sheehan \& Eisner (2017) but the code was not formally introduced at that time.

\section{Observations and Data Reduction}

Our targets were observed with ALMA Band 6 during Cycle 3, in 2016 September. We include details of each individual observation in Table 1. For each observation, the Band 6 receiver was set up with three basebands configured for wideband continuum observations, each with $2 \mathrm{GHz}$ of bandwidth and centered at 233, 246, and $248 \mathrm{GHz}$. The final baseband was configured for ${ }^{12} \mathrm{CO}$ (2-1) observations, with $38400.122 \mathrm{MHz}$ channels centered at $230.538 \mathrm{GHz}\left(0.32 \mathrm{~km} \mathrm{~s}^{-1}\right.$ velocity resolution; Hanning smoothed) in order to spectrally resolve the molecular line emission. In addition to observing our science targets, each track included time spent on quasars for the purposes of calibrating our data.

The data were calibrated using the ALMA Pipeline in the CASA software package (McMullin et al. 2007). Following the bandpass, flux, and gain calibrations, we employed a single iteration of phase-only self-calibration, with one solution per scan, to the continuum basebands and applied these phase solutions to our spectral line window. After calibration, the data were imaged and deconvolved using the spectral cube mode of the CASA clean task. The imaging was done using natural weighting to increase the sensitivity of the channel maps, as the emission is often faint, and with $0.4 \mathrm{~km} \mathrm{~s}^{-1}$ channels. We provide additional details of the images in Table 1, and for brevity we show moment 0 and 1 maps in Figure 1 and channel maps with a limited selection of channels in Figures $2-4$. We do, however, show the full channel maps in Appendix C.

We note that there is a distinct lack of redshifted emission toward DH Tau from $\sim 6-7.2 \mathrm{~km} \mathrm{~s}^{-1}$ (see Figures 1 and 4 and Appendix (), with emission reappearing at $7.4 \mathrm{~km} \mathrm{~s}^{-1}$. We suspect this lack of emission arises from extinction by 


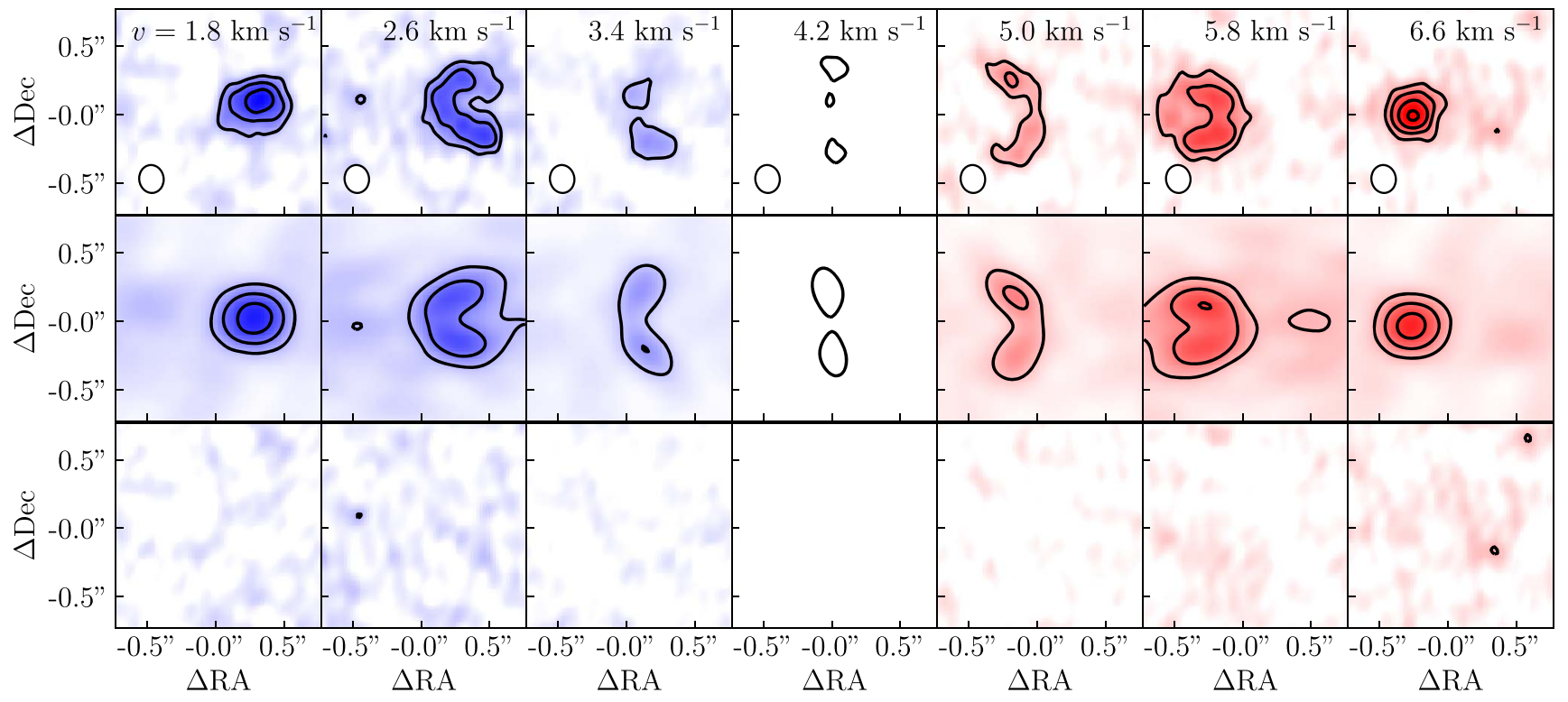

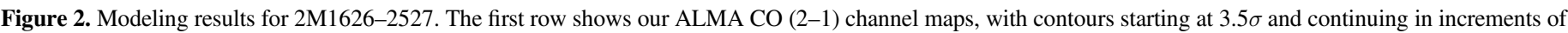

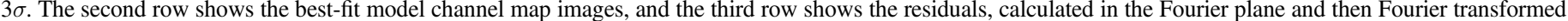
to produce an image. Here, for brevity, we show only a selection of channels, but the full channel maps can be found in Appendix C.

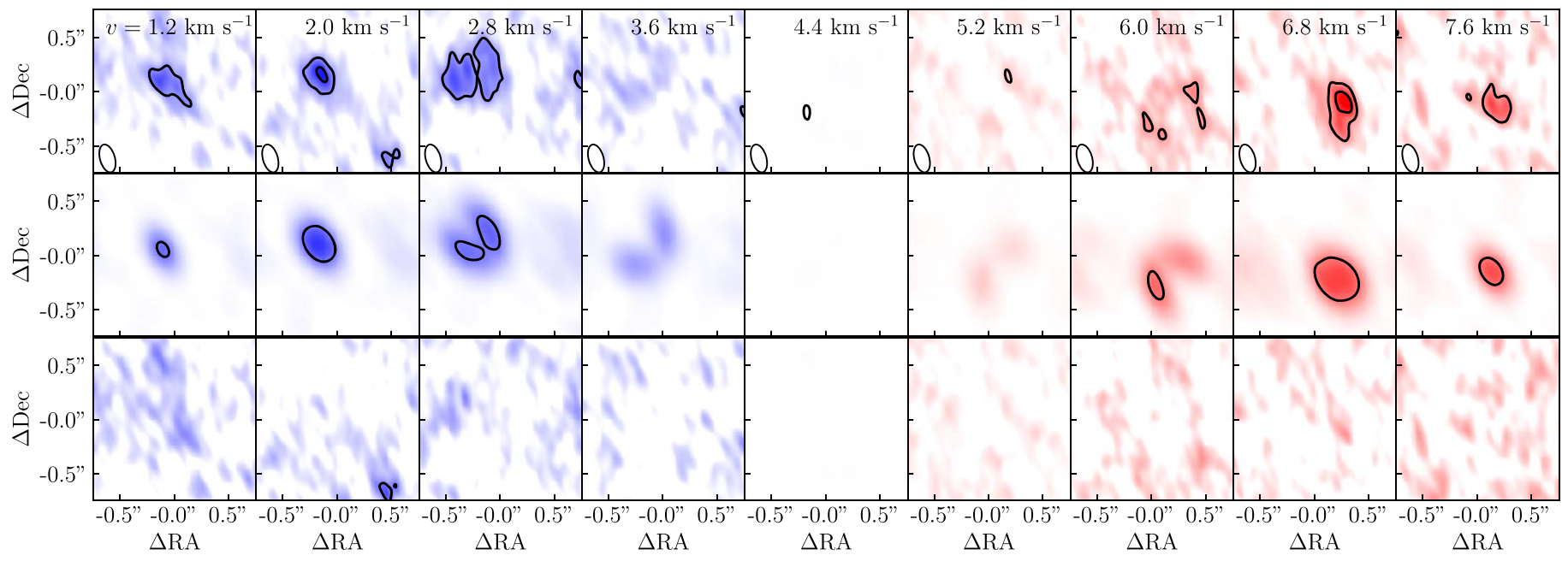

Figure 3. Best-fit model for CT Cha, including data, model, and residuals, shown in the same style as in Figure 2.

molecular cloud in that velocity range, as in the case of GO Tau (Schaefer et al. 2009). We therefore exclude those channels from the channel map modeling described in the next section. The channels that were not considered are marked with an " $X$ " in any relevant figures. A similar effect (and resolution) was adopted by Czekala et al. (2016).

\section{Analysis}

\subsection{Dynamical Stellar Masses with the pdspy Code}

Keplerian rotation imprints a unique pattern in the disk kinematics that can be seen in channel maps (the so-called "butterfly pattern"), and that pattern is strongly dependent on stellar mass. We generate synthetic Keplerian disk channel maps using radiative transfer modeling and fit these synthetic observations to the actual data using a Markov Chain Monte Carlo (MCMC) fitting routine. We describe our disk model, and our fitting procedure, in more detail below.
We assume a radial structure for our disk model that is motivated by models of viscous disk evolution (Lynden-Bell \& Pringle 1974), with the surface density given by

$$
\Sigma(r)=\Sigma_{0}\left(\frac{r}{r_{c}}\right)^{-\gamma} \exp \left[-\left(\frac{r}{r_{c}}\right)^{2-\gamma}\right]
$$

where $r$ is the stellocentric radius in cylindrical coordinates and $\gamma$ is the surface density power-law exponent. $r_{c}$ is the radius beyond which the disk surface density is exponentially tapered, and serves as a proxy for the disk radius $\left(R_{\text {disk }}=r_{c}\right)$ as the surface density profile has no hard outer limit. We assume that the disk is truncated at an inner radius of $R_{\mathrm{in}} . \Sigma_{0}$ is a proportionality constant related to the total disk gas mass,

$$
\Sigma_{0}=\frac{(2-\gamma) M_{d}}{2 \pi r_{c}^{2}}
$$




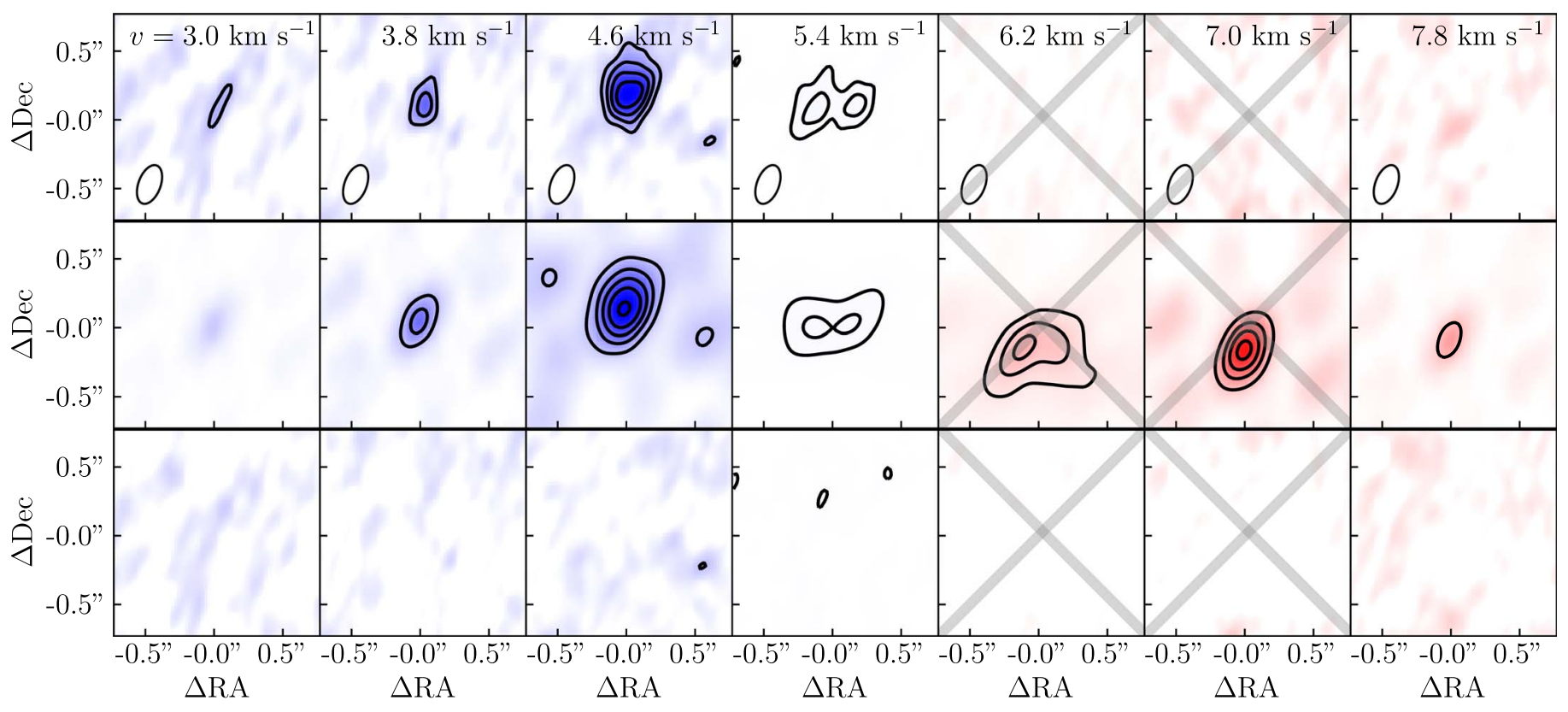

Figure 4. Best-fit model for DH Tau, including data, model, and residuals, shown in the same style as in Figure 2. The channels marked with an " $\mathrm{X}$ " show a lack of redshifted emission, likely due to absorption by a foreground cloud. The " $\mathrm{X}$ " indicates that these channels were excluded from our modeling.

As total disk gas mass is easier to interpret, we leave it as a free parameter in place of $\Sigma_{0}$.

The vertical structure of the disk is governed by hydrostatic equilibrium,

$$
\frac{\partial \log \rho}{\partial z}=-\left[\left(\frac{G M_{*} z}{\left(r^{2}+z^{2}\right)^{3 / 2}}\right)\left(\frac{\mu m_{H}}{k_{b} T_{g}}\right)+\frac{\partial \log T_{g}}{\partial z}\right],
$$

where $\rho$ is the gas density, $M_{*}$ is the stellar mass, and $T_{g}$ is the gas temperature. Moreover, $\mu=2.37$ is the mean molecular weight of the gas, appropriate for a molecular gas with solar metallicity, and $z$ is the distance in the vertical direction in cylindrical coordinates. For simplicity, we assume that the disk is vertically isothermal, with a radial temperature dependence of

$$
T_{g}(r)=T_{0}\left(\frac{r}{1 \mathrm{au}}\right)^{-q},
$$

where $T_{0}$ is the temperature at 1 au and $q$ is the temperature power-law exponent. Under these assumptions, solving the equation of hydrostatic equilibrium finds that the density structure is given by,

$$
\rho(r, z)=\frac{\Sigma(r)}{\sqrt{2 \pi} h(r)} \exp \left[-\frac{1}{2}\left(\frac{z}{h(r)}\right)^{2}\right],
$$

where $h(r)$ is the scale height of the gas. The scale height from hydrostatic equilibrium is found to be,

$$
h(r)=\left[\frac{k_{b} r^{3} T_{g}(r)}{G M_{*} \mu m_{H}}\right]^{1 / 2} .
$$

Finally, the number density of the $\mathrm{CO}$ gas can be derived from,

$$
n_{\mathrm{CO}}(r, z)=X_{\mathrm{CO}} \frac{\rho(r, z)}{\mu m_{H}},
$$

where $X_{\mathrm{CO}}$ is the abundance of $\mathrm{CO}$ relative to $\mathrm{H}_{2}$. We typically assume that $X_{\mathrm{CO}}=1.0 \times 10^{-4}$.

We also assume that the disk velocity structure is determined by Keplerian rotation, with an azimuthal velocity of,

$$
v_{\phi}(r)=\sqrt{\frac{G M_{*}}{r}} .
$$

The velocities in the vertical and radial directions are assumed to be zero. We do, however, include microturbulent line broadening $\left(\xi\right.$, in units of $\left.\mathrm{km} \mathrm{s}^{-1}\right)$ as a parameter in our fit.

This density, temperature, and velocity structure can be input into the RADMC-3D radiative transfer code (Dullemond 2012) to generate synthetic channel maps for a given set of model parameters. We assume local thermodynamic equilibrium to generate images, which is appropriate for disks as their densities are much higher than the critical density for $\mathrm{CO}$ of $\sim 10^{3} \mathrm{~cm}^{-3}$. We also leave a number of viewing orientation parameters as free parameters in our fit: inclination $(i)$, position angle (P.A.), centroid $\left(x_{0}, y_{0}\right)$, systemic velocity $\left(v_{\text {sys }}\right)$, and source distance $\left(d_{\mathrm{pc}}\right)$. The synthetic channel maps generated by ray tracing in RADMC-3D are Fourier transformed to produce synthetic visibilities, sampled in the $u v$-plane at the same baselines as our data sets, using the GALARIO code (Tazzari et al. 2017, 2018).

In all, our model includes 14 free parameters: $M_{*}, M_{\text {disk }}, R_{\text {in }}$, $R_{\mathrm{disk}}, \gamma, T_{0}, q, \xi, v_{\mathrm{sys}}, i, d_{\mathrm{pc}}$, P.A., $x_{0}$, and $y_{0}$. In practice, however, our modeling code is flexible, and allows additional model features and parameters to be included, for example, gaps in the gas density distribution, but here we restrict the model to these 14 . We provide further details on additional parameters in Appendix B.

We fit the model channel map visibilities generated by RADMC-3D and GALARIO to our data in the $u v$-plane using the MCMC fitting code emcee (Foreman-Mackey et al. 2013). For brevity, and because emcee has been broadly used by the community, we opt to provide further details on the setup of our MCMC runs, and details of the convergence in 
Table 2

Best-fit Model Parameters

\begin{tabular}{|c|c|c|c|c|c|c|c|c|c|c|c|c|}
\hline Source & $\begin{array}{c}M_{*} \\
\left(M_{\odot}\right)\end{array}$ & $\begin{array}{c}\log M_{\text {disk }} \\
\left(M_{\odot}\right)\end{array}$ & $\begin{array}{l}R_{\text {in }} \\
(\mathrm{au})\end{array}$ & $\begin{array}{l}R_{\text {disk }} \\
(\mathrm{au})\end{array}$ & $\gamma$ & $\begin{array}{c}T_{0} \\
(\mathrm{~K})\end{array}$ & $q$ & $\begin{array}{c}a_{\text {turb }} \\
\left(\mathrm{km} \mathrm{s}^{-1}\right)\end{array}$ & $\begin{array}{c}v_{\text {sys }} \\
\left(\mathrm{km} \mathrm{s}^{-1}\right)\end{array}$ & $\begin{array}{l}i \\
\left(^{\circ}\right)\end{array}$ & $\begin{array}{l}\text { P.A. } \\
\left({ }^{\circ}\right)\end{array}$ & $\begin{array}{l}d_{\mathrm{pc}} \\
(\mathrm{pc})\end{array}$ \\
\hline CT Cha & $0.796_{-0.014}^{+0.015}$ & $>-2.89$ & $<6.1$ & $9.3_{-5.1}^{+12.0}$ & $0.9_{-0.4}^{+0.2}$ & $106_{-14}^{+22}$ & $0.44_{-0.03}^{+0.04}$ & $<0.060$ & $4.608_{-0.014}^{+0.014}$ & $54.0_{-0.9}^{+1.0}$ & $49.5_{-0.6}^{+0.6}$ & $191.8_{-0.8}^{+0.7}$ \\
\hline DH Tau & $0.101_{-0.003}^{+0.004}$ & $-4.54_{-0.36}^{+0.32}$ & $<1.4$ & $<17.1$ & $1.4_{-0.2}^{+0.1}$ & $237_{-24}^{+33}$ & $0.53_{-0.04}^{+0.04}$ & $<0.187$ & $5.652_{-0.010}^{+0.009}$ & $48.4_{-1.5}^{+1.4}$ & $2.5_{-0.9}^{+0.7}$ & $135.4_{-1.2}^{+1.2}$ \\
\hline 2M1626-2527 & $0.535_{-0.007}^{+0.006}$ & $-1.07_{-0.87}^{+0.49}$ & $<4.1$ & $<25.8$ & $1.3_{-0.1}^{+0.1}$ & $57_{-4}^{+5}$ & $0.20_{-0.02}^{+0.02}$ & $0.137_{-0.010}^{+0.010}$ & $4.150_{-0.006}^{+0.005}$ & $54.0_{-0.4}^{+0.5}$ & $95.5_{-0.2}^{+0.2}$ & $138.2_{-1.3}^{+1.4}$ \\
\hline
\end{tabular}

Appendix A. The best-fit values are determined by discarding the burn-in MCMC steps and calculating the median of the sample of post-burn-in walker positions for each parameter. We also estimate the uncertainty on the measured parameters by considering the range around the median value that contains $68 \%$ of the post-burn-in walker positions. We provide further details on this process, as well as posterior probability distribution function triangle plots and plots of the walkers' steps in each parameter, in Appendix A.

\subsection{Masses and Stellar Parameters from Evolutionary Tracks}

Although masses have been previously estimated for 2M1626-2527, CT Cha, and DH Tau using evolutionary models, they were done across separate studies that do not always use uniform methodologies. As such, we find it worthwhile to repeat those measurements in a uniform way, following the method outlined in Andrews et al. (2013) and Czekala et al. (2015).

To begin, we derived the effective temperature $\left(T_{\text {eff }}\right)$ and bolometric luminosity $\left(L_{*}\right)$ by fitting optical and near-infrared photometry collected from the literature (Vrba et al. 1989; Epchtein et al. 1999; Briceño et al. 2002; Ducati 2002; Cutri et al. 2003; Monet et al. 2003; Zacharias et al. 2004; Torres et al. 2006; Robberto et al. 2012; Muiños \& Evans 2014; Henden et al. 2015; Bowler et al. 2017) with the BT-Settl atmospheric models (Allard et al. 2011) using the emcee package (Foreman-Mackey et al. 2013). For any set of input parameters, the model grid was interpolated to match $T_{\text {eff }}$ and surface gravity $(\log g)$. The overall brightness was scaled by the distance to the source $\left(d_{\mathrm{pc}}\right)$ and the radius of the star $\left(R_{*}\right)$, and then the model was reddened by some amount $\left(A_{V}\right)$ using the extinction curves from Weingartner \& Draine (2001) with $R_{V}=5.5$. We assumed a Gaussian prior on $d_{\mathrm{pc}}$ derived from Gaia parallaxes (see Appendix A). Similarly we assumed a Gaussian prior on $T_{\text {eff }}$, using temperatures collected from the literature (Herczeg \& Hillenbrand 2014; Manara et al. 2016; Bowler et al. 2017) with a standard deviation of one spectral subclass $(150 \mathrm{~K})$. Finally, the stellar luminosity $\left(L_{*}\right)$ was calculated from $T_{\text {eff }}$ and $R_{*}$.

For each post-burn in the MCMC sample, we mapped $T_{\text {eff }}$ and $L_{*}$ onto evolutionary tracks to build up a probabilistic sample of stellar masses $\left(M_{*}\right)$ and ages $\left(\tau_{*}\right)$. The stellar mass and age, and the associated uncertainties, were estimated from these samples using the median and range around the median containing $68 \%$ of the samples. This process was repeated for each of the sets of evolutionary tracks that we considered: the Baraffe et al. (2015) models, the PARSEC models (Bressan et al. 2012), and subsequent updates to low-mass $\left(<0.75 M_{\odot}\right)$ stars (Chen et al. 2014), as well as both the nonmagnetic and magnetic tracks from Feiden (2016).
Table 3

Stellar Photosphere Model Parameters and Stellar Parameters Inferred from Evolutionary Tracks

\begin{tabular}{lccc}
\hline \hline Parameters & 2M1626-2527 & CT Cha & DH Tau \\
\hline$T_{\text {eff }}$ (K) & $3746_{-122}^{+139}$ & $4402_{-166}^{+151}$ & $3628_{-65}^{+81}$ \\
$\log g$ & $3.6_{-0.7}^{+0.6}$ & $4.5_{-1.2}^{+0.7}$ & $3.1_{-0.4}^{+0.4}$ \\
$R_{*}\left(R_{\odot}\right)$ & $1.17_{-0.04}^{+0.06}$ & $2.06_{-0.05}^{+0.05}$ & $1.66_{-0.03}^{+0.04}$ \\
$d_{\text {pc }}$ (pc) & $138.3_{-1.4}^{+1.4}$ & $191.8_{-0.8}^{+0.8}$ & $135.4_{-1.3}^{+1.2}$ \\
$A_{V}$ & $2.5_{-0.6}^{+0.6}$ & $1.6_{-0.3}^{+0.3}$ & $0.5_{-0.3}^{+0.3}$ \\
$L_{*}\left(L_{\odot}\right)$ & $0.24_{-0.04}^{+0.05}$ & $1.41_{-0.16}^{+0.17}$ & $0.42_{-0.04}^{+0.04}$ \\
\hline & $M_{*}\left(M_{\odot}\right)$ & & \\
\hline Baraffe et al. (2015) & $0.53_{-0.10}^{+0.12}$ & $1.06_{-0.20}^{+0.20}$ & $0.40_{-0.03}^{+0.05}$ \\
Bressan et al. (2012) & $0.43_{-0.08}^{+0.10}$ & $0.87_{-0.17}^{+0.18}$ & $0.34_{-0.03}^{+0.04}$ \\
Chen et al. (2014) & $0.71_{-0.04}^{+0.01}$ & $0.87_{-0.13}^{+0.13}$ & $0.51_{-0.04}^{+0.05}$ \\
Feiden (2016) & $0.54_{-0.09}^{+0.12}$ & $0.90_{-0.15}^{+0.17}$ & $0.41_{-0.03}^{+0.04}$ \\
Feiden (2016) (magnetic) & $0.78_{-0.12}^{+0.11}$ & $1.41_{-0.09}^{+0.06}$ & $0.69_{-0.06}^{+0.08}$ \\
\hline & Age (Myr) & & \\
\hline Baraffe et al. (2015) & $4.41_{-1.06}^{+1.43}$ & $1.77_{-0.49}^{+0.60}$ & $1.21_{-0.10}^{+0.14}$ \\
Bressan et al. (2012) & $3.07_{-0.62}^{+0.92}$ & $1.26_{-0.32}^{+0.41}$ & $1.04_{-0.06}^{+0.07}$ \\
Chen et al. (2014) & $6.76_{-1.14}^{+1.10}$ & $1.26_{-0.21}^{+0.41}$ & $1.62_{-0.17}^{+0.19}$ \\
Feiden (2016) & $4.56_{-0.95}^{+1.41}$ & $1.41_{-0.30}^{+0.38}$ & $1.43_{-0.08}^{+0.09}$ \\
Feiden (2016) (magnetic) & $10.56_{-2.50}^{+3.15}$ & $3.51_{-0.39}^{+0.38}$ & $3.23_{-0.37}^{+0.47}$ \\
\hline
\end{tabular}

\section{Results}

We show the best-fit Keplerian disk models, as determined by our spectral line fitting procedure, to 2M1626-2527, CT Cha, and DH Tau in Figures 2-4. In these figures we show only a subset of channels to demonstrate that our models provide excellent fits to the data, but the comparison of the models to the full channel map data can be found in Appendix C. For all three sources the models provide good fits to the data, with no (or few) significant, $>3 \sigma$, residuals. The best-fit parameters found from these fits are listed in Table 2. All three disks are small, with characteristic radii of $\lesssim 15 \mathrm{au}$, although due to the exponential tapering our disk models do contain a small amount of mass at larger radii.

Table 3 lists the masses and other stellar parameters derived from the stellar photosphere models, and Figure 5 shows the best-fit models compared with the data. Figure 6 demonstrates the process of mapping $T_{\text {eff }}$ and $L_{*}$ onto the evolutionary tracks from Baraffe et al. (2015) to estimate $M_{*}$ and $\tau_{*}$. The posterior distributions for stellar mass and age for each set of evolutionary tracks are shown in Figure 7, with a gray line representing the mass we derive from our spectral line fits.

We find that the dynamical masses we measure for 2M1626-2527 and CT Cha are in reasonable agreement with masses inferred from evolutionary tracks, although we discuss the agreement for each source in more detail below. The 


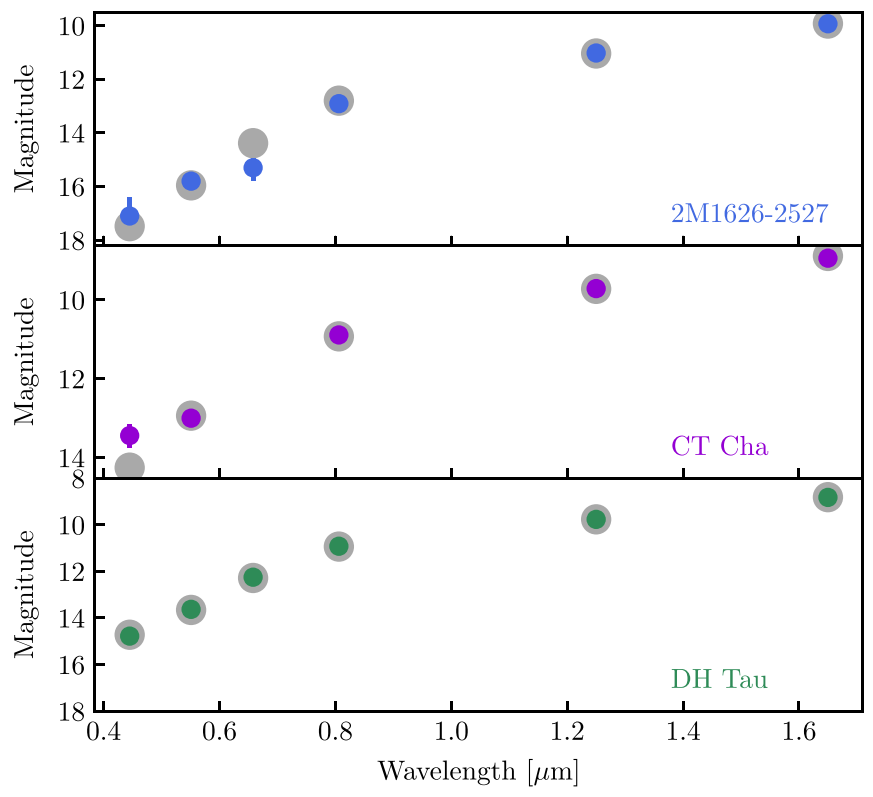

Figure 5. Optical and near-infrared photometry for our sources, with the bestfit BT-Settl model for each source shown in gray.

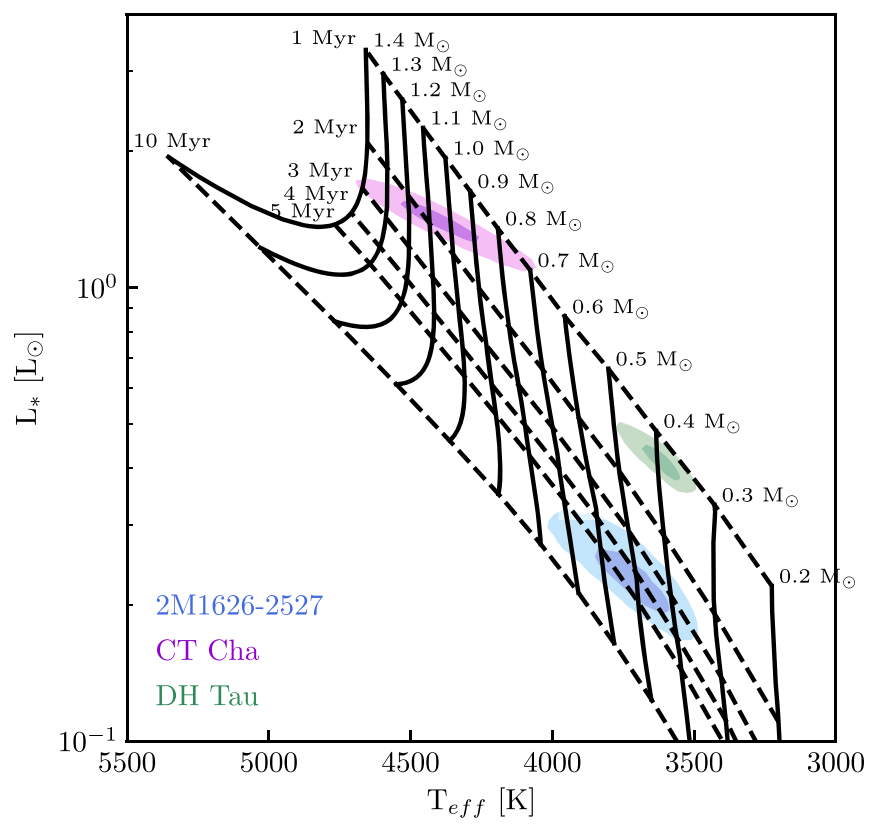

Figure 6. Pre-main-sequence evolutionary tracks from Baraffe et al. (2015) with the two-dimensional posterior probability density functions of temperature and luminosity derived from our stellar photosphere modeling. By comparing these posterior distributions with evolutionary tracks, masses and ages can be inferred.

magnetic tracks from Feiden (2016), however, seem to universally overestimate the masses for all three sources.

\section{1. $2 M 1626-2527$}

We find from our spectral line modeling that 2M1626-2527 has a dynamical mass of $0.535_{-0.007}^{+0.006} M_{\odot}$, in very good agreement with the mass estimated by the Baraffe et al. (2015) and nonmagnetic Feiden (2016) evolutionary tracks. Interestingly, though, the original PARSEC models by Bressan et al. (2012) underpredict the mass by $\sim 1 \sigma$ while the revision to the PARSEC models by Chen et al. (2014), intended to improve the tracks for stellar masses $<0.75 M_{\odot}$, overpredict 2 M1626-2527's mass by $\gtrsim 3 \sigma$. Our mass measurement is also consistent with the value of $0.5 \pm 0.1 M_{\odot}$ derived by Bowler et al. (2017), who also used the Baraffe et al. (2015) tracks.

We also find that the spin axis of 2M1626-2527 is probably not orthogonal to the disk plane (disk inclination of $54^{\circ}$ versus stellar inclination of $9^{\circ}-27^{\circ}$ found by Bowler et al. 2017). A similar misalignment of disk and star was seen in our previous study of GQ Lup (Wu et al. 2017b).

\subsection{CT Cha}

CT Cha has a stellar mass of $0.796_{-0.014}^{+0.015} M_{\odot}$, which, contrary to $2 \mathrm{M} 1626-2527$, is in very good agreement with both sets of PARSEC evolutionary tracks. The nonmagnetic Feiden (2016) tracks are also in reasonable, $\lesssim 0.5 \sigma$, agreement, while the Baraffe et al. (2015) evolutionary tracks predict a mass that is $\sim 1 \sigma$ too high. Furthermore, the mass is in reasonable $(\lesssim 0.5 \sigma)$ agreement with the mass of $0.87_{-0.18}^{+0.23} M_{\odot}$ estimated by Pascucci et al. (2016) using a combination of the Baraffe et al. (2015) and Feiden (2016) tracks.

\subsection{DH Tau}

We find that DH Tau has a mass of $0.101_{-0.003}^{+0.004} M_{\odot}$, significantly lower than the $0.3-0.5 M_{\odot}$ we derive from evolutionary track models. It is also significantly lower than the $0.2-0.5 M_{\odot}$ estimated by Hartigan et al. (1994), White \& Ghez (2001), and Andrews et al. (2013). However, it is possible that our modeling is affected by the strong molecular cloud absorption obscuring much of the low velocity emission. In order to test whether foreground extinction could be affecting our mass measurement for DH Tau, we add a simple model for the extinction to our fit. We assume that each channel is extincted by an amount of $\exp \left(-\tau_{\nu}\right)$. We assume that the optical depth, $\tau_{\nu}$, is Gaussian in shape with some central velocity, $v_{0, \text { ext }}$, width $\sigma_{\text {ext }}$, and peak value, $\tau_{0}$. We otherwise use the same model described above and fit the model to the data using the same methods, but with three additional parameters.

We find that this new model does increase the stellar mass to $0.141_{-0.011}^{+0.016} M_{\odot}$. The difference in mass can be attributed to the lower inclination found by this model, of $38.0_{-2.5}^{+2.4}$ degrees. The best-fit values of the new parameters are $v_{0, \text { ext }}=$ $6.629_{-0.018}^{+0.016} \mathrm{~km} \mathrm{~s}^{-1}, \quad \sigma_{\mathrm{ext}}=0.389_{-0.026}^{+0.027} \mathrm{~km} \mathrm{~s}^{-1}, \quad$ and $\tau_{0}=$ $6.15_{-1.01}^{+1.71}$, and all other parameters are consistent with the values we find for our base model. Although this simple model cannot fully reconcile the mass discrepancy between our measurement and the evolutionary tracks, it is possible that a more complicated extinction profile could increase the inferred stellar mass even more.

\section{Discussion}

The dynamical masses we measure from our Keplerian disk fits demonstrates the power of combining ALMA spectral line maps with precise distance measurements with Gaia. Previous Keplerian disk dynamical mass measurements exhibited extreme degeneracies between stellar mass and source distance (e.g., Czekala et al. 2015). As uncertainties in the source distance could be as large as $10 \%-20 \%$ or more, they translated directly into $10 \%-20 \%$ uncertainty on the measured stellar 

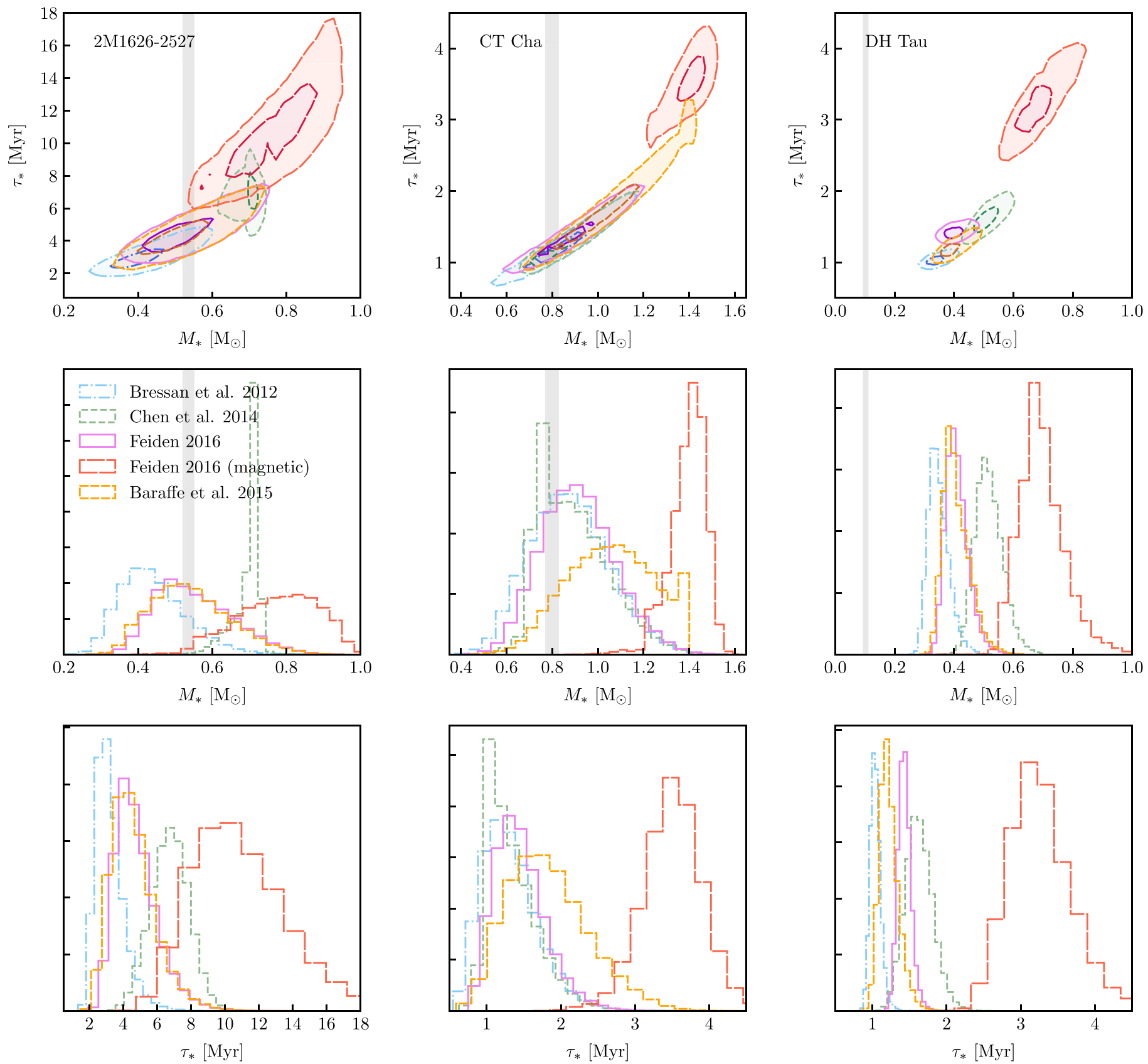

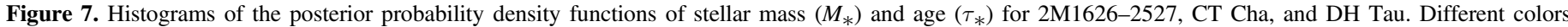

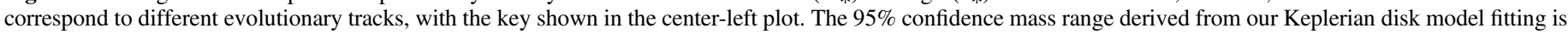
shown as a gray band.

mass. Here, using precise estimates of distances from Gaia, our stellar mass estimates have uncertainties of only $2 \%-4 \%$.

As the uncertainty on Gaia distances is $\lesssim 1 \%$, it seems likely that these uncertainties are no longer dominated by the distance uncertainty. Indeed, the posterior probability density function plots shown in Appendix A show little degeneracy between stellar mass and source distance for DH Tau and CT Cha, suggesting that the measurement is limited primarily by the quality of the ALMA data. The ALMA data for 2M1626-2527 are good enough that the Gaia distance uncertainty does affect the mass measurement, noticeable as slight a degeneracy between mass and distance, but the Gaia uncertainty is only $\sim 1 \%$ so it does not prevent us from making a high-precision measurement of the stellar mass.
Interestingly, the comparison of our dynamical mass measurements with evolutionary track mass estimates shows a wide range in agreement. The PARSEC evolutionary tracks (Bressan et al. 2012; Chen et al. 2014) do the best job of reproducing the highest mass source in our sample, CT Cha, but fail to correctly reproduce the mass of 2M1626-2527. Conversely, the Baraffe et al. (2015) evolutionary tracks do an excellent job of reproducing the mass of 2M1626-2527, but overestimate the mass of CT Cha. The nonmagnetic tracks from Feiden (2016) may provide the best balance of matching the masses of both CT Cha and 2M1626-2527, but do not match both perfectly. It is clear, though, that the magnetic tracks from Feiden (2016) do a poor job of reproducing the masses of both sources. 
The case of DH Tau is particularly interesting because the mass we measure is discrepant from evolutionary track estimates, which are uniformly $(>4 \sigma)$ too high. As we demonstrated earlier, foreground extinction could affect our stellar mass measurement, although our simple model for extinction only increases the stellar mass to $\sim 0.14 M_{\odot}$ and does not reconcile with evolutionary tracks. Of course, a more complicated extinction profile may increase the stellar mass by more. If the mass estimate is correct, however, then a relatively major shift in the evolutionary tracks for young, low-mass objects is needed. Higher signal-to-noise data may help us to better understand the effects of cloud contamination on this source and better constrain its mass, as might more optically thin gas tracers that better see through the foreground cloud. For now, however, it remains unclear whether the discrepancy is a result of the limitations of the data, or whether there are real problems with the evolutionary tracks.

Moving forward, however, a much larger sample of premain-sequence stars is needed to understand whether the trends suggested by our targets are more generally true, and thereby truly place constraints on evolutionary tracks. Our results here demonstrate that with relatively modest observations $(\sim 15$ minutes on source; 0 "! $2-0$ "! 3 resolution; $0.4 \mathrm{~km} \mathrm{~s}^{-1}$ channels) we can robustly measure stellar masses as low as $\sim 0.1 M_{\odot}$ Moreover, with channel widths as small as $0.03-0.05 \mathrm{~km} \mathrm{~s}^{-1}$ for $\mathrm{CO}(2-1)$ or $\mathrm{CO}(3-2)$, it should be possible to directly measure masses for young substellar and planetary-mass objects. Building a sample with several tens of pre-mainsequence sources could therefore reasonably be done.

\section{Conclusions}

We have used our Keplerian disk radiative transfer code psdpy to fit ALMA ${ }^{12} \mathrm{CO}(2-1)$ channel maps for three $\mathrm{T}$ Tauri stars 2M1626-2527, CT Cha, and DH Tau. With new, precise distance measurements for these sources from Gaia, we are able to make high-precision $(\sim 2 \%-4 \%)$ direct measurements of their masses. The mass uncertainty is no longer dominated by distance as in previous studies, but instead by the quality of ALMA data. We find that 2M1626-2527 has a mass of $0.535_{-0.007}^{+0.006} M_{\odot}$, CT Cha has a mass of $0.796_{-0.014}^{+0.015} M_{\odot}$, and DH Tau has a mass of $0.101_{-0.003}^{+0.004} M_{\odot}$. Comparing with the stellar masses estimated using multiple sets of evolutionary tracks, we find that both 2M1626-2527 and CT Cha are in reasonable agreement with most models $(<2 \sigma)$, although potentially significant differences remain for most sets of tracks. The large discrepancy for DH Tau may be caused by a significant local cloud obscuration, which completely absorbs the redshifted disk emission, although simple models of the foreground extinction only increase the mass to $0.141_{-0.011}^{+0.016} M_{\odot}$.

Looking toward the future, the synergy of ALMA, Gaia, and detailed radiative transfer disk modeling will enable precise mass measurements of a large number of pre-main-sequence stars, and even for substellar objects, and ultimately place stringent constraints on evolutionary models.

This paper makes use of the following ALMA data: ADS/ JAO.ALMA\#2015.1.00773.S. ALMA is a partnership of ESO (representing its member states), NSF (USA) and NINS (Japan), together with NRC (Canada), NSC and ASIAA (Taiwan), and KASI (Republic of Korea), in cooperation with the Republic of Chile. The Joint ALMA Observatory is operated by ESO, AUI/NRAO, and NAOJ. The National
Radio Astronomy Observatory is a facility of the National Science Foundation operated under cooperative agreement by Associated Universities, Inc. Y.-L. W. is grateful to the support from the Heising-Simons Foundation. J.T. would like to acknowledge support from the Homer L. Dodge Endowed Chair. This material is based upon work supported by the National Science Foundation Graduate Research Fellowship under grant No. 2012115762. This work was supported by NSF AAG grant 1311910. The results reported herein benefitted from collaborations and/or information exchange within NASA's Nexus for Exoplanet System Science (NExSS) research coordination network sponsored by NASA's Science Mission Directorate. The computing for this project was performed at the OU Supercomputing Center for Education and Research (OSCER) at the University of Oklahoma (OU).

Software: pdspy (Sheehan 2018), CASA (McMullin et al. 2007), RADMC-3D (Dullemond 2012), emcee (Foreman-Mackey et al. 2013), matplotlib (Hunter 2007), corner (Foreman-Mackey 2016), GALARIO (Tazzari et al. 2018).

\section{Appendix A The MCMC Fitting Procedure}

We fit our radiative transfer model, as described in Section 3.1, to our data using the MCMC fitting code emcee (Foreman-Mackey et al. 2013). emcee uses an implemenation of the Goodman \& Weare (2010) Affine-Invariant ensemble sampler to sample parameter space with a large number of walkers. We use 200 separate MCMC "walkers," which we spread out over a large range of parameter space. These walkers are allowed to move through parameter space, with their steps being toward another randomly selected walker and the size of the step dictated by a comparison of the goodness of fit of each set of walker parameters. The likelihood function for a given set of parameters $\hat{\theta}$ used for the fit is given by

$$
\begin{aligned}
\ell(\hat{\theta})= & \exp \left[-\frac{1}{2} \sum_{i=1}^{N} \sum_{j=1}^{M} \mid V\left(u_{i}, v_{i}, \nu_{j}\right)\right. \\
& \left.-\left.M\left(u_{i}, v_{i}, \nu_{j} \mid \hat{\theta}\right)\right|^{2} W\left(u_{i}, v_{i}, \nu_{j}\right)\right]
\end{aligned}
$$

where $V\left(u_{i}, v_{i}, \nu_{j}\right)$ are the complex visibility data at baseline $\left(u_{i}, v_{i}\right)$ and frequency $\nu_{j}, W\left(u_{i}, v_{i}, \nu_{j}\right)$ are the visibility data weights, and $M\left(u_{i}, v_{i}, \nu_{j} \mid \hat{\theta}\right)$ is the model with parameters $\hat{\theta}$ evaluated at baseline $\left(u_{i}, v_{i}\right)$ and frequency $\nu_{j} . N$ is the total number of baselines in the visibility data, and $M$ is the number of channels included in the fit. We consider a fit to be "converged" when the median walker position does not change significantly compared to the spread of the walkers over a significant number of steps. We show the steps taken by the walkers in each fit in Figures 8-10.

We note that while we commonly refer to the 14 parameters of our model fit as $\hat{\theta}=\left\{M_{*}, M_{\mathrm{disk}}, R_{\mathrm{in}}, R_{\mathrm{disk}}, \gamma, T_{0}, q, \xi, v_{\mathrm{sys}}, i, d_{\mathrm{pc}}\right.$, P.A., $\left.x_{0}, y_{0}\right\}$, reasonable ranges of values for a number of these parameters span multiple orders of magnitude. For such parameters, it makes practical sense to use the log of the parameter as the actual fit quantity. For this reason, the actual parameters being fit are $\hat{\theta}=\left\{\log _{10} M_{*}, \log _{10} M_{\mathrm{disk}}, \log _{10} R_{\mathrm{in}}, \log _{10} R_{\mathrm{disk}}, \gamma, \log _{10} T_{0}, q\right.$, $\log _{10} \xi, v_{\mathrm{sys}}, i, d_{\mathrm{pc}}$, P.A., $\left.x_{0}, y_{0}\right\}$. As the linear values are more intuitive to understand; however, for most tables and figures and discussion in the text, we convert the posterior MCMC samples to linear values before evaluating best-fit values. We assume a 

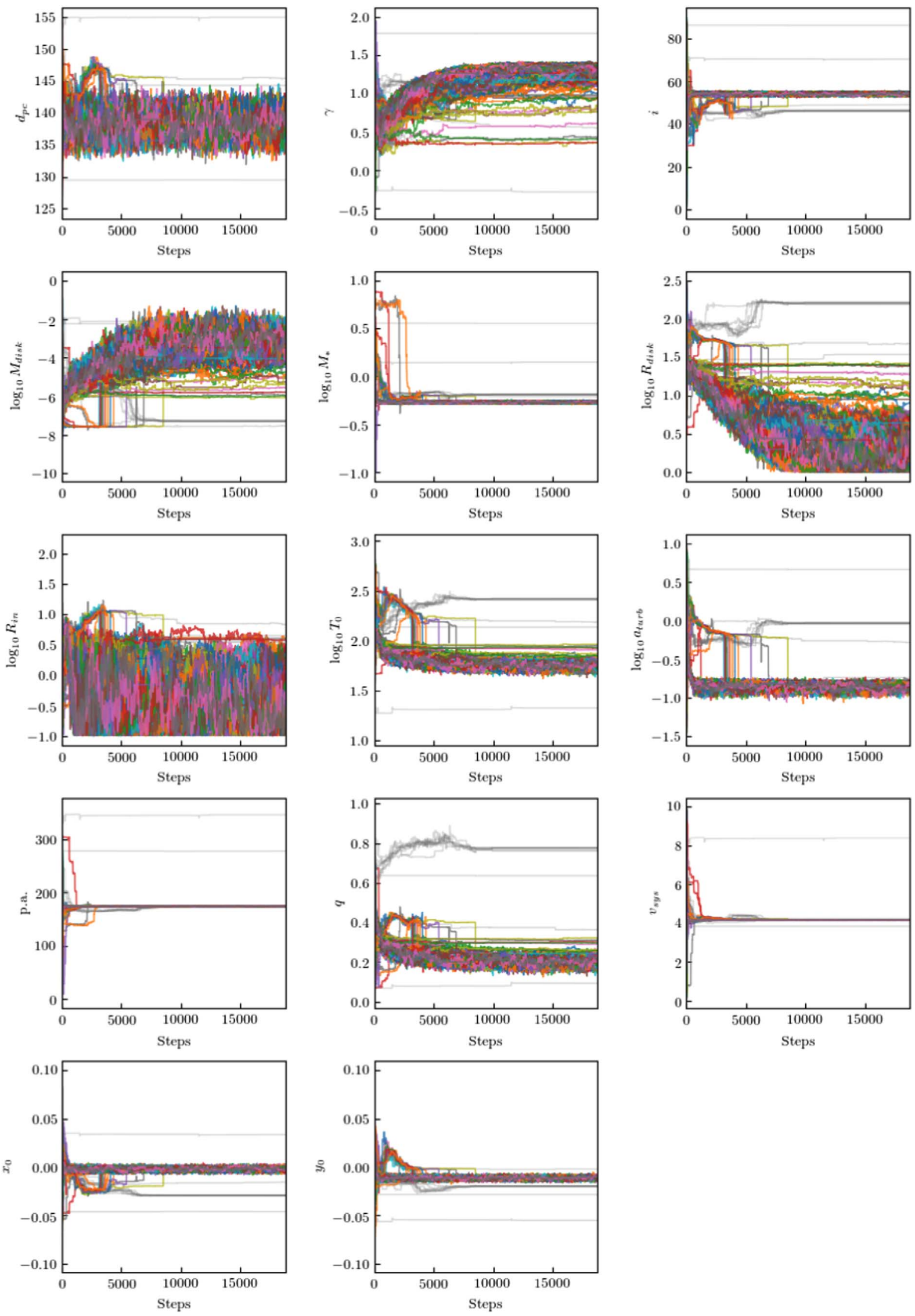

Figure 8. Steps taken by the MCMC walkers in the fit to 2M1626-2527. The "lost" walkers are shown in gray. 

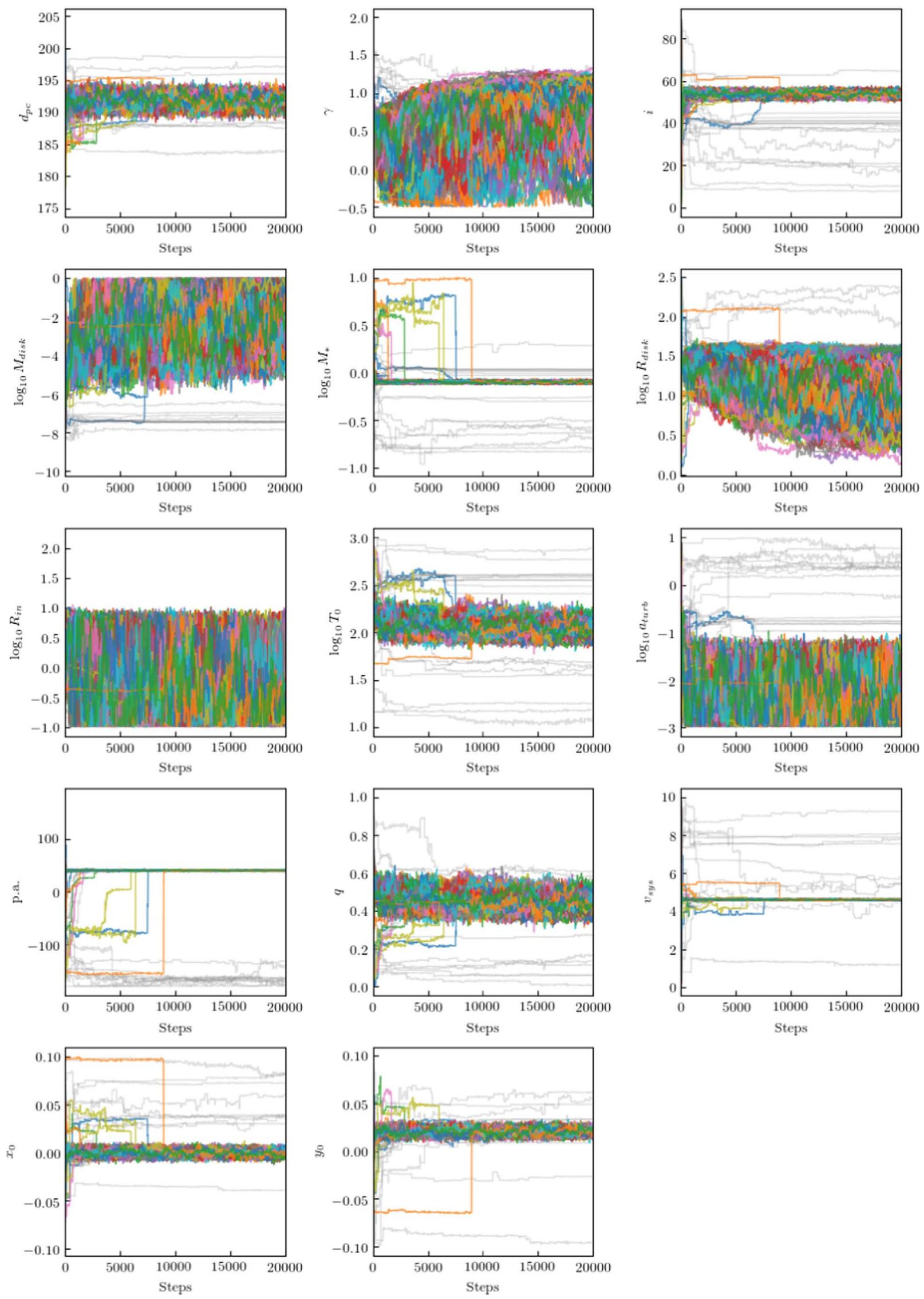

Figure 9. Steps taken by the MCMC walkers in the fit to CT Cha. The "lost" walkers are shown in gray. 

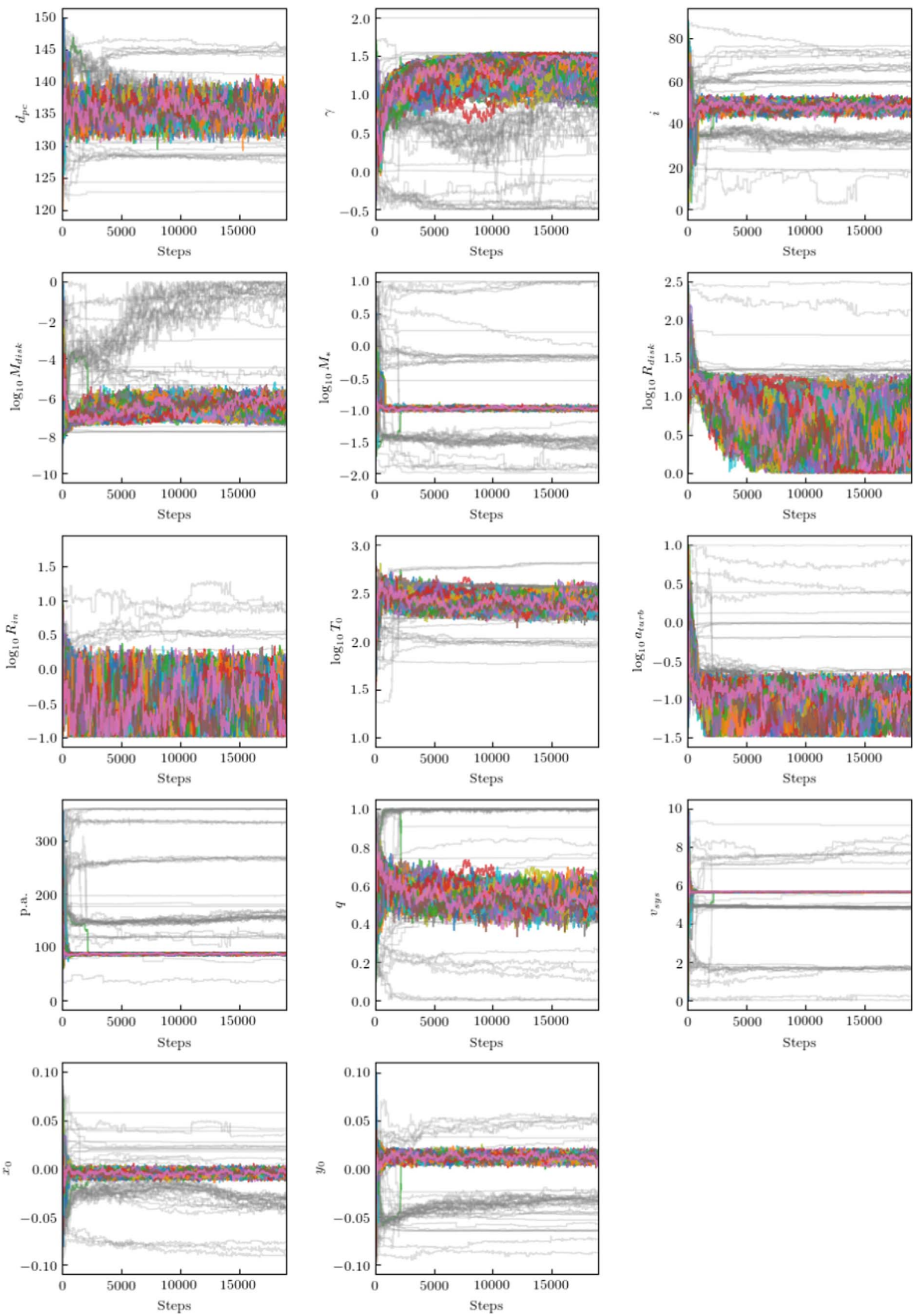

Figure 10. Steps taken by the MCMC walkers in the fit to DH Tau. The "lost" walkers are shown in gray. 
uniform prior on all parameters except for the source distance $\left(d_{\mathrm{pc}}\right)$ and stellar mass $\left(M_{*}\right)$, with the following limits: $-2 \leqslant \log _{10} M_{\text {star }} \leqslant 1,-10 \leqslant \log _{10} M_{\text {disk }} \leqslant 0,-1 \leqslant \log _{10} R_{\text {in }} \leqslant$ $1.5, \quad 0 \leqslant \log _{10} R_{\text {disk }} \leqslant 2.5, \quad-0.5 \leqslant \gamma \leqslant 2, \quad 1 \leqslant \log _{10} T_{0} \leqslant 3$, $0 \leqslant q \leqslant 1,-1.5 \leqslant \log _{10} a_{\text {turb }} \leqslant 1,0^{\circ} \leqslant i \leqslant 90^{\circ}, 0^{\circ} \leqslant$ p.a. $\leqslant$ $360^{\circ},-0$." $1 \leqslant x_{0} \leqslant 0$ ! $1,-0$ !' $1 \leqslant y_{0} \leqslant 0$ ". 1 . In principle, sensitive ALMA spectral line data can distinguish between inclinations above and below $90^{\circ}$ (e.g., Czekala et al. 2015). However our initial tests found that the data does not have the required sensitivity, with the walkers split approximately evenly between the two solutions, so we limit $i<90^{\circ}$.

For a young star for which the stellar mass is not a priori known, the probability of that star having mass $M_{*}$ is given by the stellar initial mass function. As such we use the Chabrier IMF (Chabrier 2003) as a prior on the stellar mass in our fit. Furthermore, while $d_{\mathrm{pc}}$ is left as a free parameter in the fit, our ALMA data do not provide a direct constraint on its value. As stellar mass is highly degenerate with source distance (e.g., Czekala et al. 2015), placing some constraint on source distance is important. Moreover, strong constraints on source distance lead to higher precision measurements of stellar mass. Here, we use direct measurements of source distances from trigonometric parallaxes by Gaia to place a prior constraint on the distance. We find from Gaia that 2M1626-2527 has a parallax of 7.232 \pm 0.074 mas and a distance of $138.258_{-1.396}^{+1.426} \mathrm{pc}$, CT Cha has a parallax of $5.214 \pm 0.021$ mas and a distance of $191.775_{-0.774}^{+0.781} \mathrm{pc}$, and DH Tau has a parallax of $7.388 \pm 0.069$ mas and a distance of $135.355_{-1.258}^{+1.282}$ pc (Gaia Collaboration et al. 2018). We assume a Gaussian prior on the parallax, with a mean given by the measured Gaia parallax and the standard deviation given by the Gaia uncertainty. The model parallax is calculated as $1 / d_{\mathrm{pc}}$.

While in general the walkers tend to converge into one bunch, a handful of walkers appear to become lost in parameter space and have trouble finding their way to the main group (e.g., see Figure 8). We believe that this is the result of how the walkers move through parameter space. To determine the next step for a walker, a different walker is randomly drawn from the sample of walkers. The walker will (randomly) move toward or away from the randomly selected walker with a step size between $1 / a$ and $a$ times the distance between them ( $a=2$ by default in emcee). The walkers become "lost," however, because the global minimum is quite narrow in some parameters (e.g., $v_{\text {sys }}$ ), so the likelihood that the step puts it into the main bunch (i.e., $a=1$ ) becomes very small. It therefore takes a large number of step proposals before the walker finally makes the jump. While we have confidence that, if given enough time, these walkers would converge as well, the computational demands of these fits are significant, so we opt not to continue running the fits. Instead, we discard any walkers with $\left(\chi^{2}-\chi_{\min }^{2}\right)>44$, appropriate for a $\chi^{2}$ distribution with 14 parameters and $p=0.00006(\sim 4 \sigma)$. The autocorrelation time for these fits is typically about $\sim 1000$ steps, so with 5000 post-burn-in steps in the chain, we have $\sim 5$ independent sets of walker positions. With 200 walkers, or a total of $\sim 1000$ independent samples, we would expect $\ll 1(\sim 0.06)$ walkers to have $\left(\chi^{2}-\chi_{\min }^{2}\right)>44$, so this cut will identify any "lost" walkers that are not likely representative of the underlying distribution. We show the steps taken by all walkers in Figures 8-10. The discarded walkers shown in gray to demonstrate that the cuts are reasonable.

In Figures 11-13 we show the two-dimensional posterior probability density functions for each pair of parameters, after the burn-in steps and "lost" walkers have been discarded, for each source. We estimate the best-fit value for a parameter as the median of the walker positions in that parameter after convergence is achieved, the "lost" walkers are discarded, and the walker steps are trimmed by the autocorrelation time. Similarly, we estimate the uncertainties on that value as the region around the median containing $68 \%$ of the walker positions after convergence and "lost" walkers are discarded. For parameters where the walkers move up against an edge of the allowed range ( $a_{\text {turb }}$ for CT Cha and DH Tau, $R_{\text {disk }}$ for DH Tau and 2M1626-2527, $M_{\text {disk }}$ for CT Cha, $R_{\text {in }}$ for all), we instead use the value containing $99.7 \%$ of walkers above or below that value as a limit. 


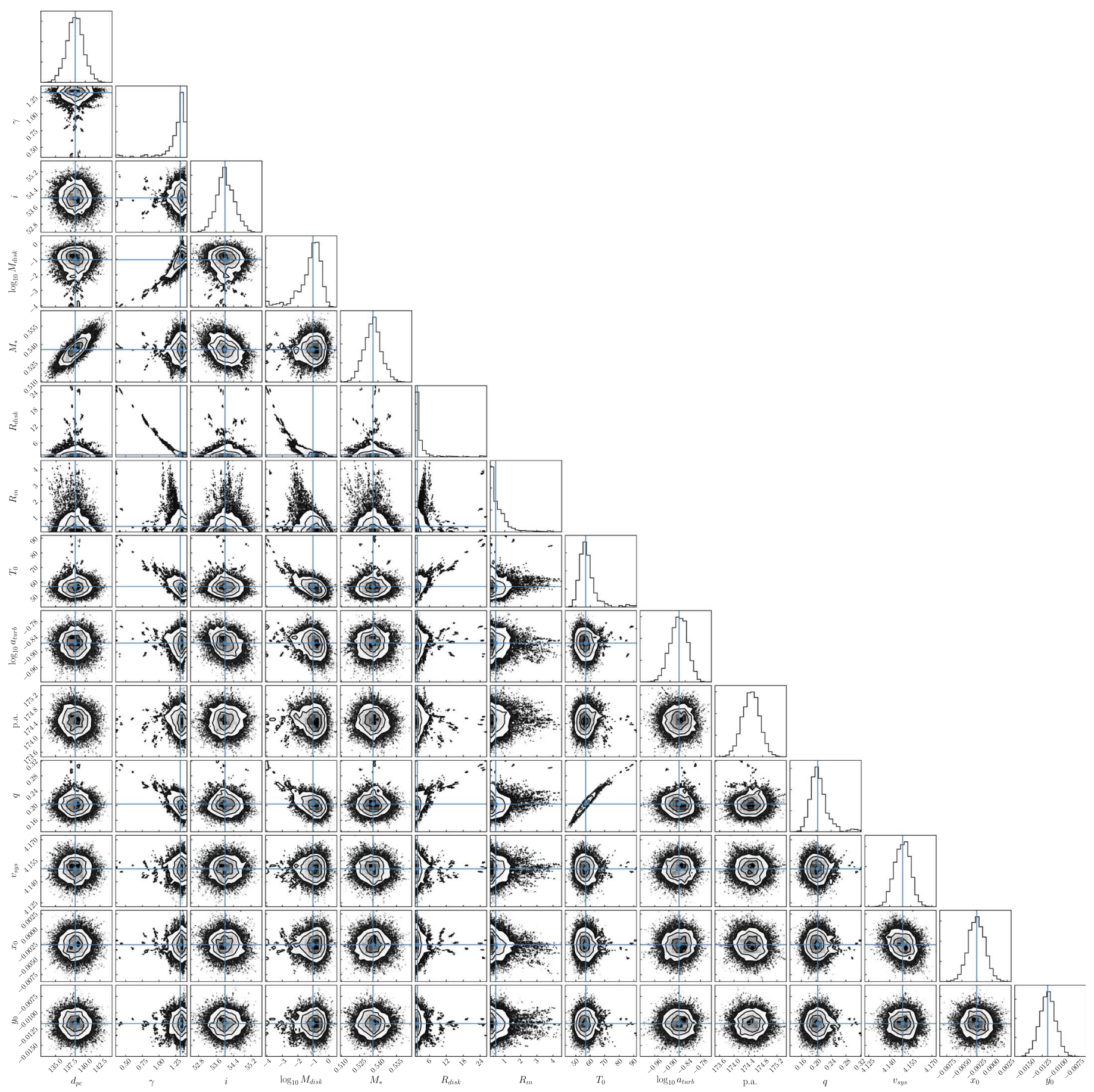

Figure 11. One- and two-dimensional posterior probability density functions for the fit to 2M1626-2527. The best-fit values are shown as horizontal and vertical lines. 


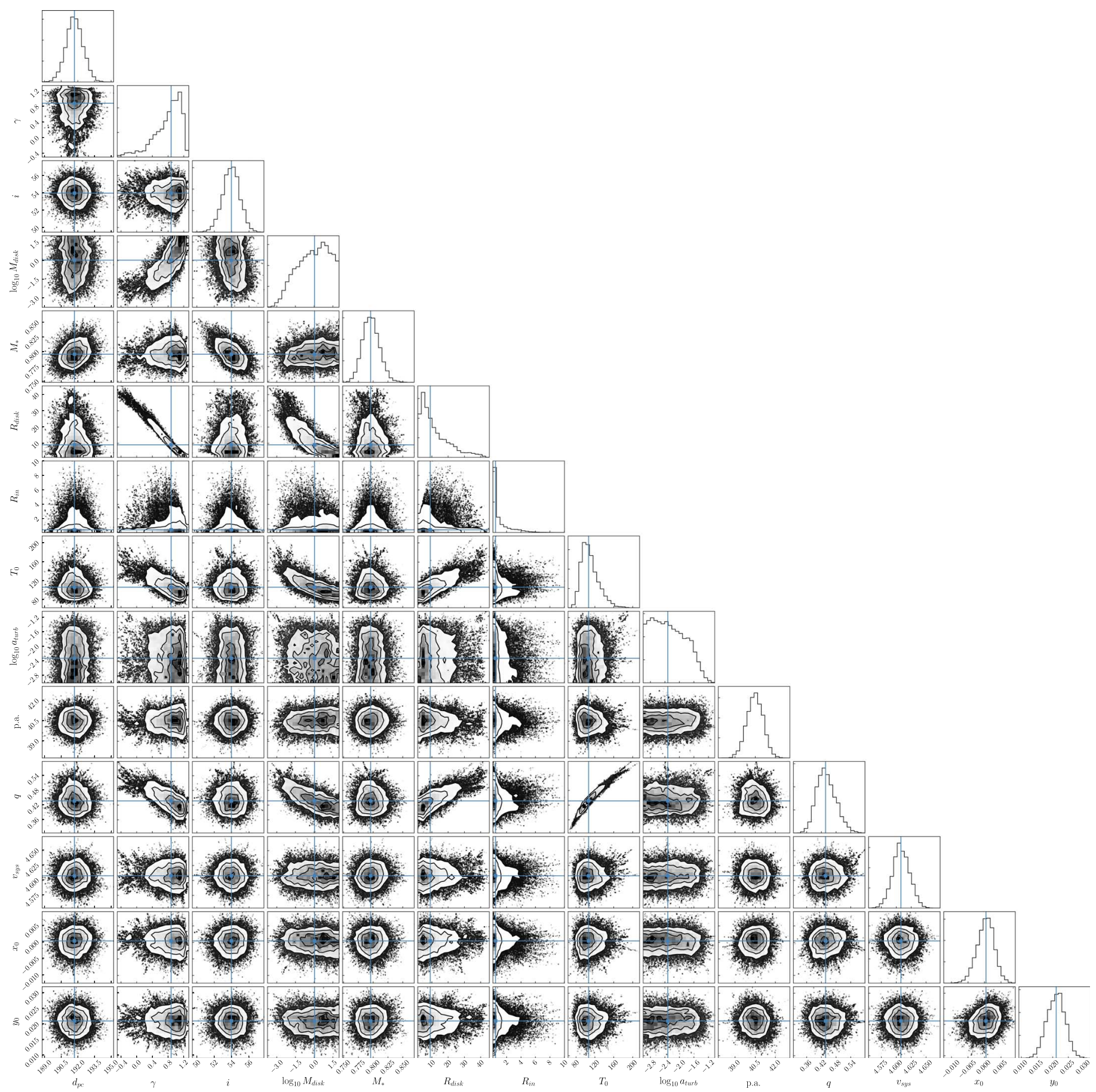

Figure 12. One- and two-dimensional posterior probability density functions for the fit to CT Cha. The best-fit values are shown as horizontal and vertical lines. 


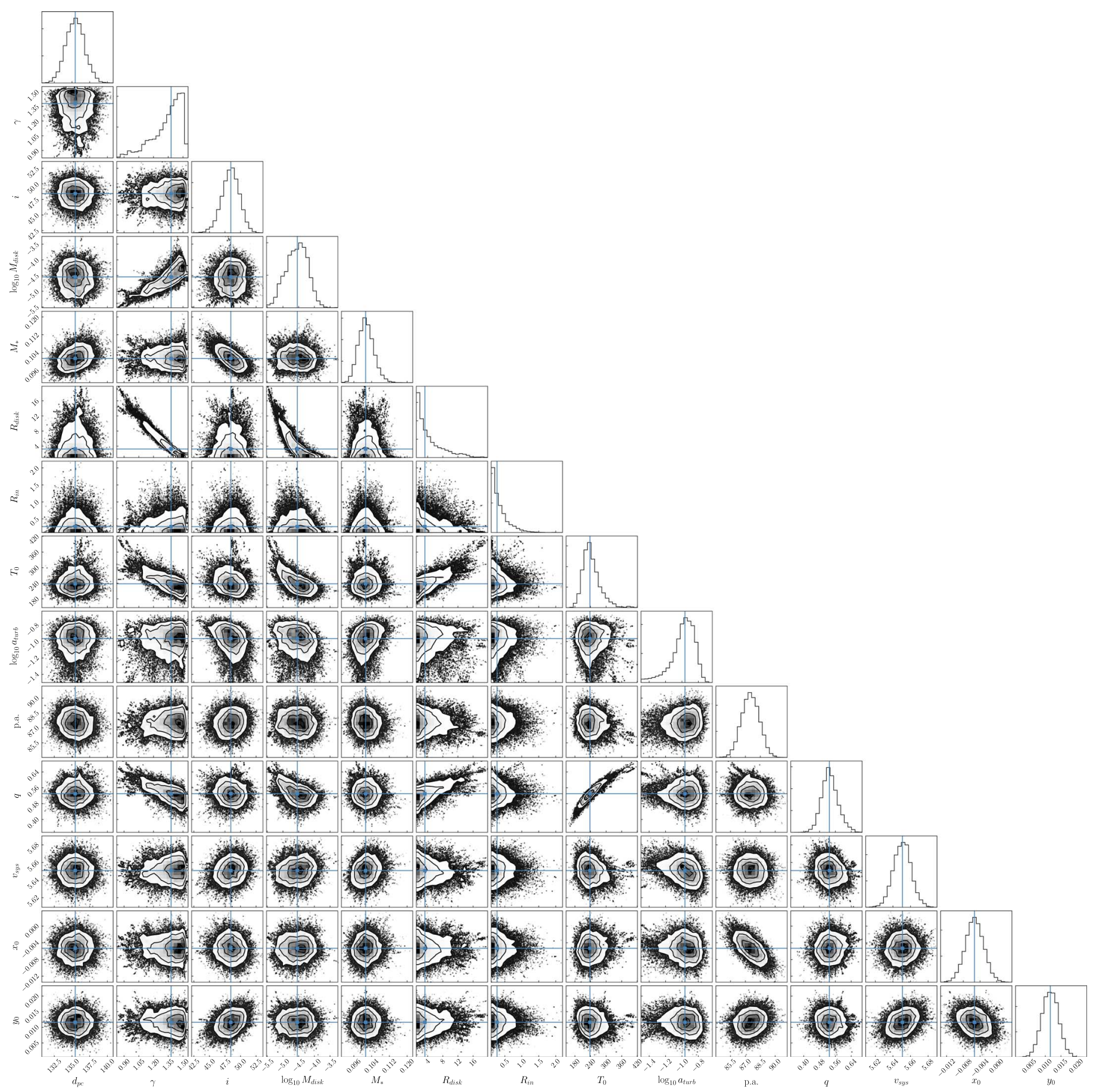

Figure 13. One- and two-dimensional posterior probability density functions for the fit to DH Tau. The best-fit values are shown as horizontal and vertical lines. 


\section{Appendix B Additional Model Parameters}

pdspy is designed to be a flexible modeling tool that can handle a range of input parameters, priors, and models. For example, here we have left the surface density profile powerlaw exponent, $\gamma=1$, as a free parameter. However, the walker plots show that $\gamma$ converges slowly and does not ultimately affect the measured value of $M_{*}$. As such, it may be wise to fix $\gamma=1$ (e.g., Czekala et al. 2015) to reduce the needed computational time, and this can easily be done in pdspy by changing a single boolean value in the configuration file. This, of course, may not be wise if the goal is to measure disk structure, as parameters like $M_{\text {disk }}$ and $R_{\text {disk }}$ are degenerate with $\gamma$, but for studies measuring stellar mass this would be appropriate. In addition to adding or removing parameters from our model, pdspy also includes a variety of models that can be employed. Here we have employed an exponentially tapered surface density profile; however, pdspy includes options for a truncated disk model as well. Moreover, up to three gaps and a cavity can be added as features of the model. Finally, simple Gaussian priors can be added to any parameter, and a few "special" priors are included in the code. As described above the distance can be constrained with a prior on the parallax, and the stellar mass can be constrained by using the IMF as a prior.

In all, pdspy currently has over 25 parameters that can be turned on or off at the users discretion, as well as options to use a truncated disk and to include continuum opacity/subtraction. We are also exploring adding additional functionality, including nonvertically isothermal disk temperatures and inclusion of envelope emission for embedded protostars. More information, including the current, up-to-date status of modeling options, can be found at https://github.com/psheehan/pdspy.

\section{Appendix C \\ Channel Map Images and Models}

In Figures 14-16, we show the best-fit models and residuals for each of our sources, here including every channel in the maps. 

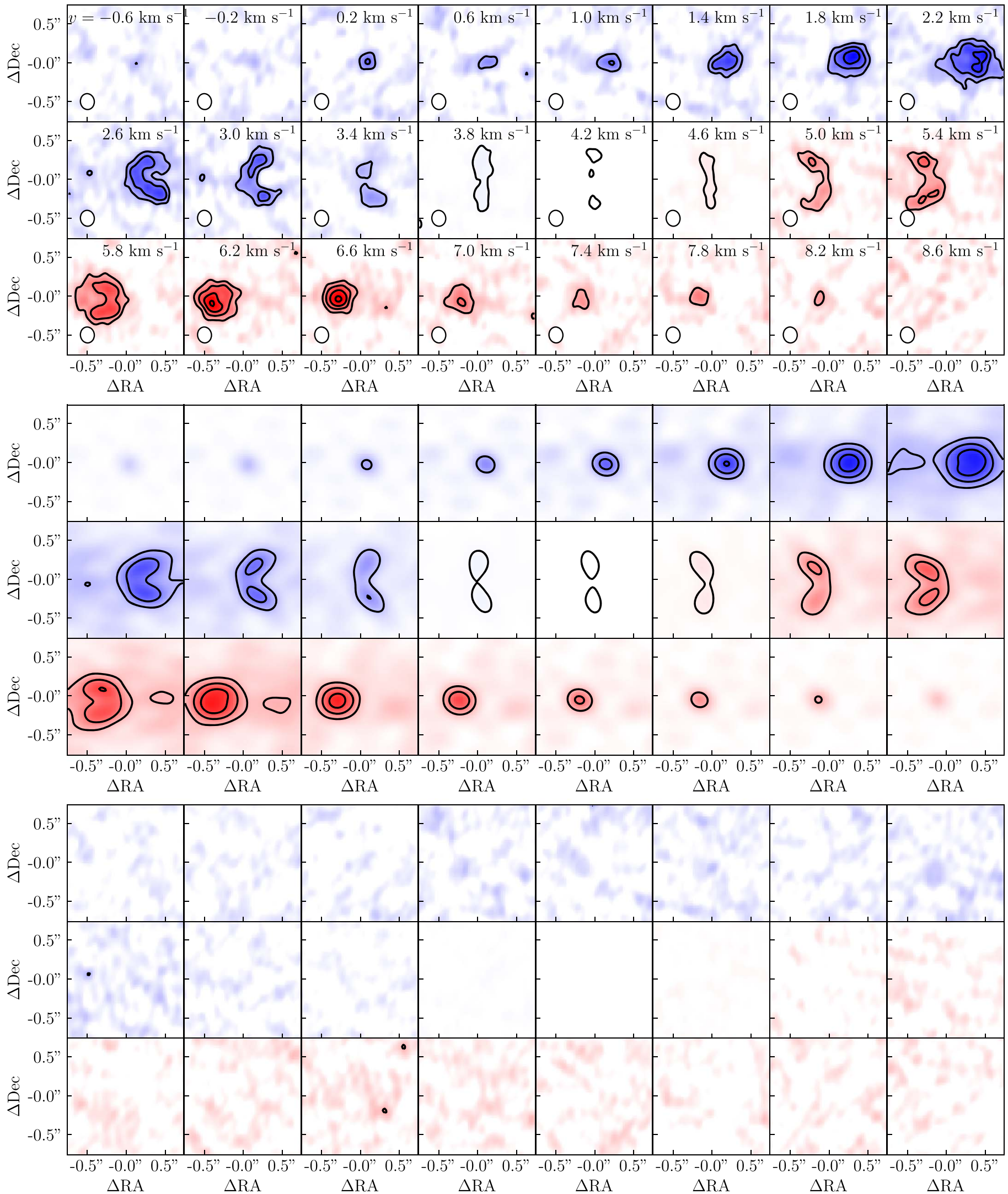

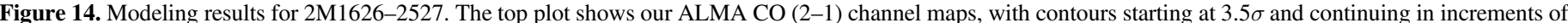

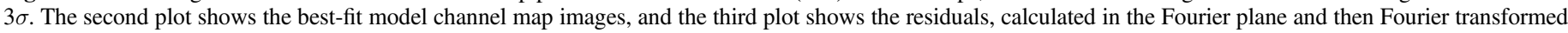
to produce an image. 

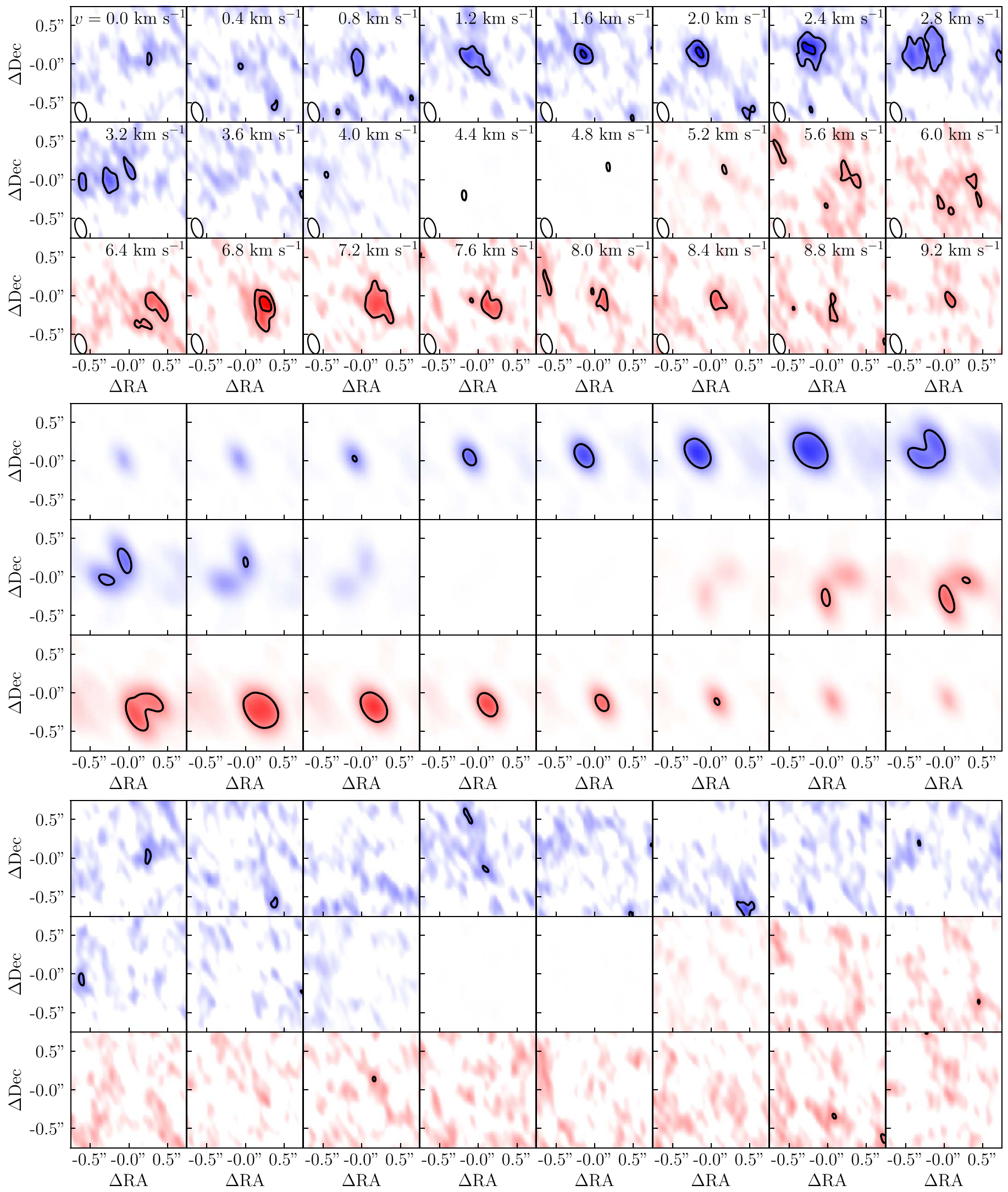

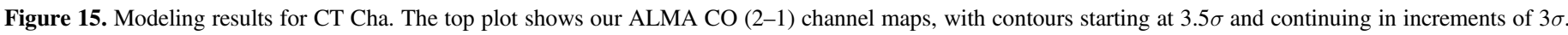

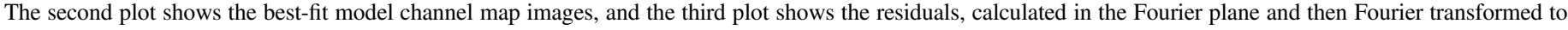
produce an image. 

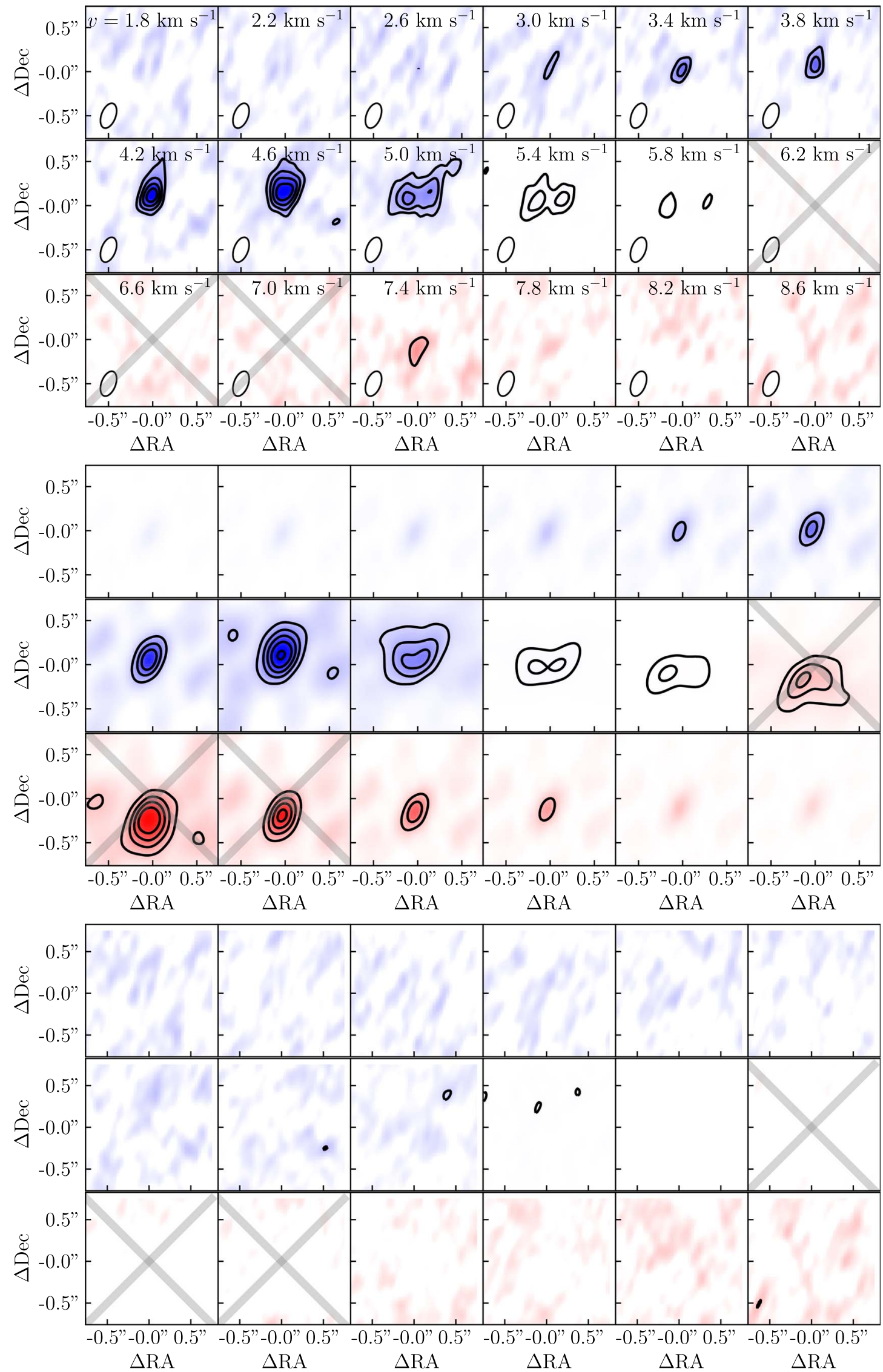

Figure 16. Modeling results for DH Tau. The top plot shows our ALMA CO (2-1) channel maps, with contours starting at $3.5 \sigma$ and continuing in increments of $3 \sigma$. The second plot shows the best-fit model channel map images, and the third plot shows the residuals, calculated in the Fourier plane and then Fourier transformed to produce an image. The channels marked with an " $\mathrm{X}$ " show a lack of redshifted emission, likely due to absorption by a foreground cloud. The " $\mathrm{X}$ " indicates that these channels were excluded from our modeling. 


\section{ORCID iDs}

Patrick D. Sheehan (1) https://orcid.org/0000-0002-9209-8708

Ya-Lin Wu (1) https://orcid.org/0000-0002-4392-1446

Josh A. Eisner (i) https://orcid.org/0000-0002-1031-4199

John J. Tobin (i) https://orcid.org/0000-0002-6195-0152

\section{References}

Allard, F., Homeier, D., \& Freytag, B. 2011, in ASP Conf. Ser. 448, XVI Cambridge Workshop on Cool Stars, Stellar Systems, and the Sun, ed. C. Johns-Krull, M. K. Browning, \& A. A. West (San Francisco, CA: ASP), 91

Andrews, S. M., Rosenfeld, K. A., Kraus, A. L., \& Wilner, D. J. 2013, ApJ, 771,129

Ansdell, M., Williams, J. P., Trapman, L., et al. 2018, ApJ, 859, 21

Ansdell, M., Williams, J. P., van der Marel, N., et al. 2016, ApJ, 828, 46

Baraffe, I., Homeier, D., Allard, F., \& Chabrier, G. 2015, A\&A, 577, A42

Barenfeld, S. A., Carpenter, J. M., Ricci, L., \& Isella, A. 2016, ApJ, 827, 142

Bell, C. P. M. 2016, in 19th Cambridge Workshop on Cool Stars, Stellar Systems, and the Sun (CS19), 102, Zenodo, doi:10.5281/zenodo.58936

Bowler, B. P., Kraus, A. L., Bryan, M. L., et al. 2017, AJ, 154, 165

Bressan, A., Marigo, P., Girardi, L., et al. 2012, MNRAS, 427, 127

Briceño, C., Luhman, K. L., Hartmann, L., Stauffer, J. R., \& Kirkpatrick, J. D. 2002, ApJ, 580, 317

Chabrier, G. 2003, PASP, 115, 763

Chen, Y., Girardi, L., Bressan, A., et al. 2014, MNRAS, 444, 2525

Cutri, R. M., Skrutskie, M. F., van Dyk, S., et al. 2003, yCat, 2246, 0

Czekala, I., Andrews, S. M., Jensen, E. L. N., et al. 2015, ApJ, 806, 154

Czekala, I., Andrews, S. M., Torres, G., et al. 2016, ApJ, 818, 156

Czekala, I., Andrews, S. M., Torres, G., et al. 2017, ApJ, 851, 132

Ducati, J. R. 2002, yCat, 2237, 0

Dullemond, C. P. 2012, RADMC-3D: A Multi-purpose Radiative Transfer Tool, Astrophysics Source Code Library, ascl:1202.015

Dupuy, T. J., Forbrich, J., Rizzuto, A., et al. 2016, ApJ, 827, 23

Dutrey, A., Guilloteau, S., \& Simon, M. 1994, A\&A, 286, 149

Dutrey, A., Guilloteau, S., \& Simon, M. 2003, A\&A, 402, 1003

Eisner, J. A., Arce, H. G., Ballering, N. P., et al. 2018, ApJ, 860, 77

Epchtein, N., Deul, E., Derriere, S., et al. 1999, A\&A, 349, 236

Feiden, G. A. 2016, A\&A, 593, A99

Flaherty, K. M., Hughes, A. M., Rose, S. C., et al. 2017, ApJ, 843, 150

Flaherty, K. M., Hughes, A. M., Rosenfeld, K. A., et al. 2015, ApJ, 813, 99

Flaherty, K. M., Hughes, A. M., Teague, R., et al. 2018, ApJ, 856, 117

Foreman-Mackey, D. 2016, JOSS, 1, 24

Foreman-Mackey, D., Hogg, D. W., Lang, D., \& Goodman, J. 2013, PASP, 125,306

Gaia Collaboration, Brown, A. G. A., Vallenari, A., et al. 2018, A\&A, 616, A1

Gennaro, M., Prada Moroni, P. G., \& Tognelli, E. 2012, MNRAS, 420, 986

Goodman, J., \& Weare, J. 2010, Communications in Applied Mathematics and Computational Science, 5, 65

Haisch, K. E., Jr., Lada, E. A., \& Lada, C. J. 2001, ApJL, 553, L153
Hartigan, P., Strom, K. M., \& Strom, S. E. 1994, ApJ, 427, 961

Henden, A. A., Templeton, M., Terrell, D., et al. 2015, AAS Meeting, 225, 336.16

Herczeg, G. J., \& Hillenbrand, L. A. 2014, ApJ, 786, 97

Hernández, J., Hartmann, L., Calvet, N., et al. 2008, ApJ, 686, 1195

Hillenbrand, L. A., \& White, R. J. 2004, ApJ, 604, 741

Hunter, J. D. 2007, CSE, 9, 90

Lynden-Bell, D., \& Pringle, J. E. 1974, MNRAS, 168, 603

MacGregor, M. A., Wilner, D. J., Czekala, I., et al. 2017, ApJ, 835, 17

Manara, C. F., Fedele, D., Herczeg, G. J., \& Teixeira, P. S. 2016, A\&A, 585, A136

McMullin, J. P., Waters, B., Schiebel, D., Young, W., \& Golap, K. 2007, in ASP Conf. Ser. 376, Astronomical Data Analysis Software and Systems XVI, ed. R. A. Shaw, F. Hill, \& D. J. Bell (San Francisco, CA: ASP), 127

Miotello, A., van Dishoeck, E. F., Williams, J. P., et al. 2017, A\&A, 599, A113

Monet, D. G., Levine, S. E., Canzian, B., et al. 2003, AJ, 125, 984

Muiños, J. L., \& Evans, D. W. 2014, AN, 335, 367

Öberg, K. I., Furuya, K., Loomis, R., et al. 2015, ApJ, 810, 112

Ortiz-León, G. N., Loinard, L., Kounkel, M. A., et al. 2017, ApJ, 834, 141

Pascucci, I., Testi, L., Herczeg, G. J., et al. 2016, ApJ, 831, 125

Ricci, L., Cazzoletti, P., Czekala, I., et al. 2017, AJ, 154, 24

Rizzuto, A. C., Ireland, M. J., Dupuy, T. J., \& Kraus, A. L. 2016, ApJ, 817,164

Robberto, M., Spina, L., Da Rio, N., et al. 2012, AJ, 144, 83

Rodet, L., Bonnefoy, M., Durkan, S., et al. 2018, A\&A, 618, A23

Schaefer, G. H., Dutrey, A., Guilloteau, S., Simon, M., \& White, R. J. 2009, ApJ, 701, 698

Schwarz, K. R., Bergin, E. A., Cleeves, L. I., et al. 2016, ApJ, 823, 91

Sheehan, P. 2018, psheehan/pdspy: pdspy: A MCMC Tool for Continuum and Spectral Line Radiative Transfer Modeling, Zenodo, doi:10.5281/zenodo. 2455079

Sheehan, P. D., \& Eisner, J. A. 2017, ApJ, 851, 45

Simon, M., Dutrey, A., \& Guilloteau, S. 2000, ApJ, 545, 1034

Simon, M., Guilloteau, S., Di Folco, E., et al. 2017, ApJ, 844, 158

Stassun, K. G., Feiden, G. A., \& Torres, G. 2014, NewAR, 60, 1

Tazzari, M., Beaujean, F., \& Testi, L. 2017, Galario: Gpu Accelerated Library for Analyzing Radio Interferometer Observations, Astrophysics Source Code Library, ascl: 1710.022

Tazzari, M., Beaujean, F., \& Testi, L. 2018, MNRAS, 476, 4527

Torres, C. A. O., Quast, G. R., da Silva, L., et al. 2006, A\&A, 460, 695

Vrba, F. J., Rydgren, A. E., Chugainov, P. F., Shakovskaia, N. I., \& Weaver, W. B. 1989, AJ, 97, 483

Ward-Duong, K., Patience, J., Bulger, J., et al. 2018, AJ, 155, 54

Weingartner, J. C., \& Draine, B. T. 2001, ApJ, 548, 296

White, R. J., \& Ghez, A. M. 2001, ApJ, 556, 265

Williams, J. P., \& Best, W. M. J. 2014, ApJ, 788, 59

Wu, Y.-L., Close, L. M., Eisner, J. A., \& Sheehan, P. D. 2017a, AJ, 154, 234

Wu, Y.-L., \& Sheehan, P. D. 2017, ApJL, 846, L26

Wu, Y.-L., Sheehan, P. D., Males, J. R., et al. 2017b, ApJ, 836, 223

Zacharias, N., Urban, S. E., Zacharias, M. I., et al. 2004, AJ, 127, 3043 